\title{
Ultrasound Biomicroscopy in Glaucoma: An Update
}

\author{
Tanuj Dada, Gaurav Kumar, Sanjay Kumar Mishra
}

Associate Professor, Glaucoma Facility, Dr RP Center for Ophthalmic Sciences, All India Institute of Medical Sciences New Delhi, India

\begin{abstract}
UBM is a high-frequency ultrasound technology that provides exceptionally detailed two-dimensional gray-scale images of the various anterior segment structures and evaluates them both quantitatively and qualitatively. It allows noninvasive in vivo imaging of structural details of the anterior ocular segment at near microscopic resolution and thus, there are many other applications of this new imaging method.This review outlines the uses of UBM in diagnosis and management of glaucoma.
\end{abstract}

\section{INTRODUCTION}

Ultrasound Biomicroscopy (UBM) is a high resolution ultrasound technique developed by Pavlin, Sherar and Foster in Toronto in the late 1980s. UBM is a high-frequency ultrasound technology that allows noninvasive in vivo imaging of structural details of the anterior ocular segment at near microscopic resolution. It provides exceptionally detailed two-dimensional gray-scale images of the various anterior segment structures and evaluates them both quantitatively and qualitatively. There are many other applications of this new imaging method. Examples of other uses include imaging adnexal pathology, assessing corneal changes with refractive surgery, the assessment of trauma, and determination of intraocular lens position.

\section{PRINCIPLE}

UBM uses a scan transducer having a high frequency. The transducer frequency of conventional diagnostic ultrasound instruments is in the range of 7.5 to $10 \mathrm{MHz}$. In contrast, the transducer frequency of the UBM instrument is approximately $50 \mathrm{MHz}^{1-3}$

UBM provides much higher image resolution (approximately $25 \mu \mathrm{m}$ of axial and $50 \mu \mathrm{m}$ of lateral resolution) than does conventional B-scan ocular ultrasonography. The improved imaged resolution is attributable to the higher transducer frequency of the UBM.

UBM is not able to image as deeply into the eye as is conventional B-scan. This is because improved image resolution comes at the expense of reduced depth of penetration of the ultrasonic beam (limited to approximately $5 \mathrm{~mm}$ for a $50 \mathrm{MHz}$
UBM instrument). The limited depth of penetration is also associated with a smaller angular field.

The real-time image is displayed on a video monitor and can be recorded. The room illumination, fixation and accommodative effort of the patient should be held constant particularly while doing quantitative evaluation.

\section{TECHNIQUE}

The image acquisition technique is similar to traditional immersion B-scan ultrasonography. The examination is done with the patient in the supine position, after local anesthetic has been applied to the eye. A sufficient palpebral fissure must be present to accommodate an eye cup (plastic or silicone) which is used to create a small water bath. Normally $1 \%$ or $2 \%$ methylcellulose solution is used as the coupling fluid. Kapetansky FM et $\mathrm{al}^{4}$ have proposed the use of a water bath that fits the eye so well that saline, a superior coupling agent, can be substituted for methylcellulose. This new water bath is a flexible polysiloxane cup with a beveled inner edge, providing a watertight seal and thus permitting the use of saline as the coupling medium for UBM. Saline, because of its lack of impurities, generates images that are superior in quality to those produced with methylcellulose as the coupling medium.

The eye cup does cause some discomfort, limiting the utility of this technique in children and some anxious adults. While doing the scan, one should not give undue pressure on the eye cup as it can distort the angle structures. Scanning is performed with the suspended arm of the instrument held above the eye cup and with the ultrasound transducer oscillating within the methylcellulose solution. The transducer should be oriented so that the scanning beam strikes the target perpendicularly, to maximize the detection of reflected signals.

Esaki et $\mathrm{al}^{5}$ have developed a reliable method to perform UBM in the prone and sitting positions with no loss of image quality. This method can expand the ability of the UBM to examine alterations in anatomic relationships among anterior segment structures between the supine, sitting, and prone positions.

Software within the instrument is designed to stop the instrument if it comes too close to the cornea, thus preventing corneal damage. 


\section{NORMAL OCULAR STRUCTURES}

Before analyzing the UBM images of pathological eye it is essential to understand UBM imaging of normal eye. The normal anterior segment structures are given in Figures 1 to 5.

\section{CLINICAL USES IN GLAUCOMA}

\section{Qualitative Ultrasound Biomicroscopy}

\section{Examination of the Anterior Chamber Angle}

With the UBM, one can examine the angle structures in which iris, ciliary body, and scleral spur can be recognized easily. The scleral spur is the only constant landmark allowing one to interpret images and is the key for analyzing angle pathology. The scleral spur can be identified in the region where the radiopaque shadow of the sclera merges with the relatively radiolucent shadow of the cornea. ${ }^{6}$

\section{Biometry of the Anterior Segment}

With the UBM one can determine the corneal thickness, anterior chamber depth, posterior chamber depth, IOL thickness, iris thickness, ciliary body thickness, scleral thickness, etc. However, one cannot determine the lens thickness due to the reduced depth of penetration $(<5 \mathrm{~mm})$, thereby not allowing visualization of the posterior capsule of the lens. ${ }^{7}$

\section{Determining the Mechanism of Primary Glaucoma}

Ultrasound biomicroscopy is usually able to determine the mechanism of elevated intraocular pressure (angle closure versus open angle) by showing the relationship between the peripheral iris and the trabecular meshwork. ${ }^{6}$ In addition, imaging of the anterior segment structures is possible even in eyes with corneal edema or corneal opacification that precludes gonioscopic assessment.

In open-angle glaucoma, UBM can be used to measure the anterior chamber angle in degrees, to assess the configuration of the peripheral iris, and to evaluate the iris insertion in relation to the trabecular meshwork.

In eyes with a narrow angle, the mean chamber depth (ACD) in primary angle closure eye is approximately $1.8 \mathrm{~mm}$ which is $1 \mathrm{~mm}$ shorter than in normal eyes. Angle closure becomes a rarity when anterior chamber depth exceed $2.5 \mathrm{~mm}{ }^{8}$ Primary angle closure can be due to papillary block, angle crowding (from plateau iris configuration or anterior lens position) or a combination. UBM shows the extent of angle closure, reveals the depth of the anterior and posterior chambers, and identifies pathologic processes pushing the lens and iris forward.

Sihota and Dada performed UBM studies in the subtypes of primary angle closure glaucoma ${ }^{9}$ and documented that eyes with primary angle closure glaucoma have a thinner iris and shorter trabecular ciliary process distance in addition to a narrow angle. Acute primary angle closure eyes have the narrowest angle recess. In eyes with primary angle closure, older age and a shallower ACD appear to be important causes of increased forward bowing of the iris ${ }^{10}$ resulting in pupillary block. Thus UBM can predict the risk of developing angle closure.

Angle crowding is one of the mechanism of PAC, it can coexist with pupillary block. It can be described as the sandwiching of peripheral iris between the trabecular meshwork and some other structures, compared to pupillary block related anterior iris shift due to pressure differential between anterior

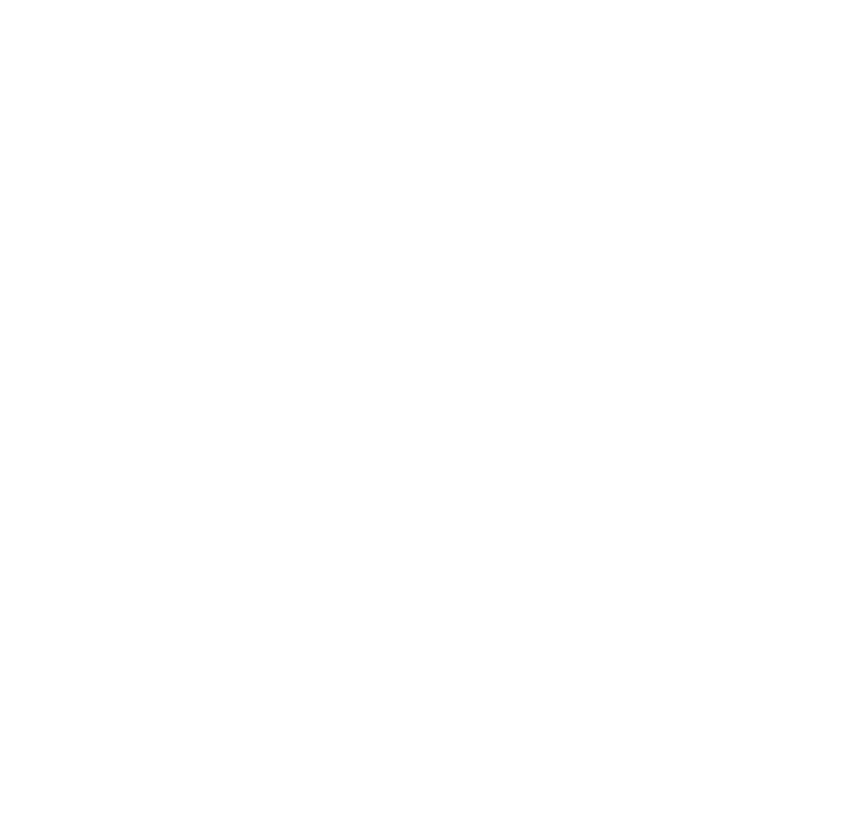

Fig. 1: Anterior chamber

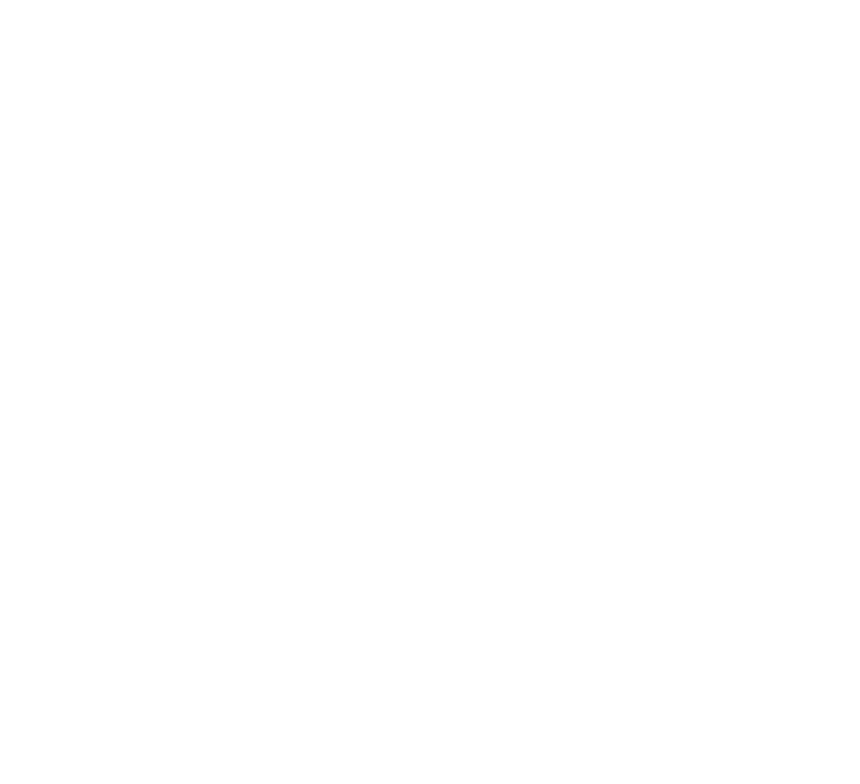

Fig. 2: Angle-open 


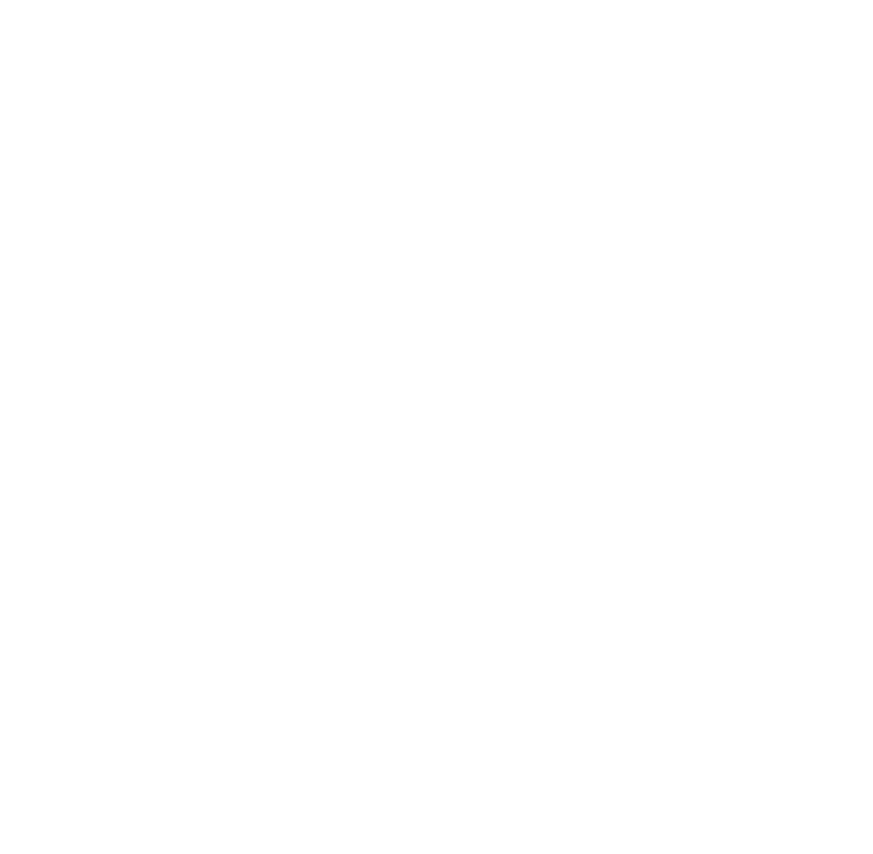

Fig. 3: Zonules

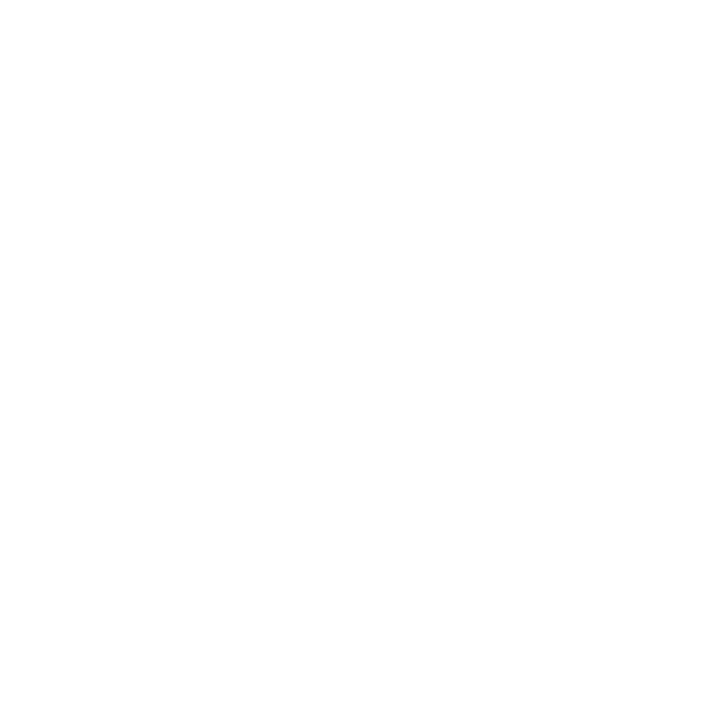

Fig. 4: Ciliary process

and posterior chamber. Plateau iris is one such primary condition. ${ }^{11}$

Plateau iris has been defined based on UBM by Kumar et al. ${ }^{12}$ Plateau iris was defined if all criteria fulfilled in at least 2 quadrants:

1. The ciliary process was anteriorly directed, supporting the peripheral iris so that it was parallel to the trabecular meshwork.

2. The iris root had a steep rise from its point of insertion, followed by a downward angulation from the corneoscleral wall.

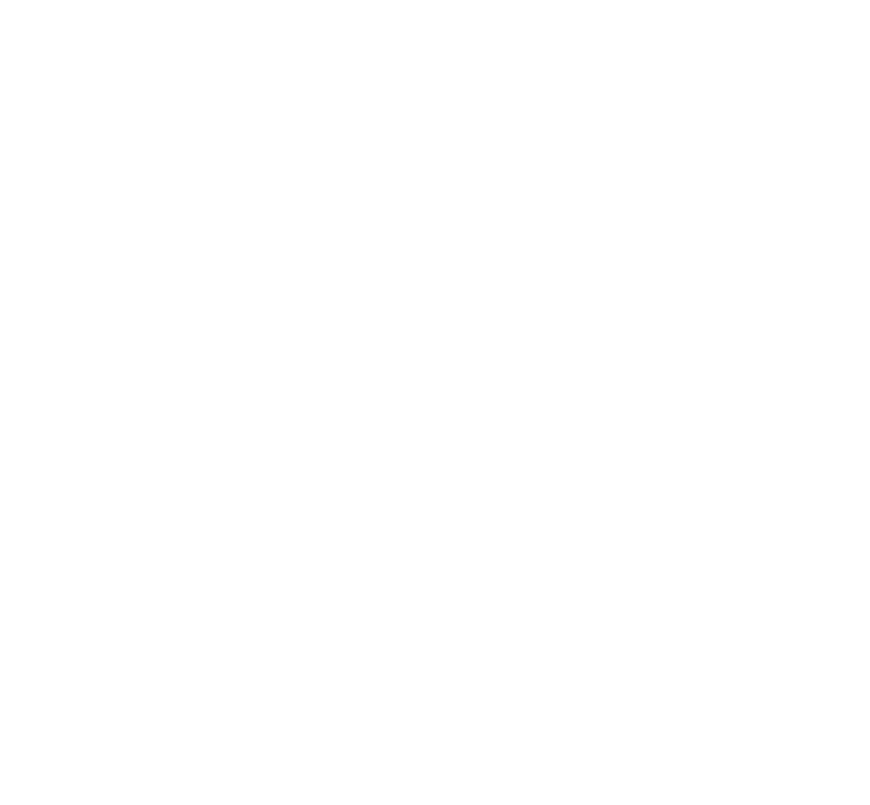

Fig. 5: Pars plana

3. Presence of a central flat iris plane.

4. An absent ciliary sulcus.

5. Irido-angle contact (above the level of the scleral spur) in the same quadrant.

In plateau iris syndrome, UBM usually reveals an abnormally steep anterior angulation of the peripheral iris, anterior insertion of the iris on to the anterior ciliary body, and retroiridic projection of the ciliary processes. It can also confirm the double hump sign which is normally seen with gonioscopy by use of an indentation UBM, a special technique that imposes mild pressure on peripheral cornea with the skirt of eyecup. ${ }^{13}$

Matsunaga $\mathrm{K}$ et al ${ }^{14}$ studied Indentation UBM gonioscopy. They found that the angle of all examined eyes was significantly widened with indentation. The angle changes in eyes with relative pupillary block (RPB) were significantly greater than in eyes with peripheral anterior synechiae (PAS) or plateau iris configuration (PIC). It was concluded that Indentation UBM gonioscopy is a very useful method for observing the angle and diagnosis of RPB, PAS, and PIC.

Garudadri CS et al ${ }^{15}$ evaluated the presence of plateau iris in eyes with primary angle-closure glaucoma (PACG) after laser peripheral iridotomy by gonioscopy and ultrasound biomicroscopy and the pathogenesis of this condition by comparing the UBM parameters of these eyes with those in normal subjects. Among the PACG eyes, after YAG iridectomy; $40 \%$ had an open angle (angle opening distance $>130$ microns) and $60 \%$ eyes had a narrow angle (angle opening distance $<$ or $=130$ microns). A large anteriorly placed ciliary process with a narrow ciliary sulcus was found in $40.9 \%$ eyes with open angle, and $66.66 \%$ eyes with narrow angles. Trabecular ciliary process 
distance was significantly larger in the eyes with open angles compared with those with narrow angles. Anteriorly directed ciliary processes were seen both in eyes with plateau iris as well as in eyes with PACG that had deep anterior chambers after iridotomy. Thus plateau iris could be picked up on UBM and found to be common in the study population.

Argon laser iridoplasty is an effective and safe treatment for plateau iris syndrome and may also prove valuable in the treatment of plateau-like iris configuration resulting from iridociliary cysts. ${ }^{16,17}$

\section{To Determine Occludability of the Angle}

One can perform dark room provocative testing with the UBM, to study the spontaneous occlusion of the angle under conditions of decreased illumination (Figs 6 and 7). This helps to identify "at risk" population which can then be subjected to a laser iridotomy. It is better than dark room gonioscopy because the latter is time consuming and standardization of slit-lamp illumination is difficult. ${ }^{6}$

Wang $\mathrm{N}$ et al ${ }^{18}$ compared the traditional dark room provocative tests (gonioscopy and applanation tonometry) with UBM biometry. With the appositional angle closure as a positive diagnostic criterion, the sensitivity of the traditional test and UBM dark room test was $31.8 \%$ and $68.2 \%$, respectively. The specificity of both methods is $100 \%$. UBM dark room provocative test elevates the sensitivity and specificity of traditional dark room test, and reduces the false negative or positive rate in screening PACG, that is helpful in its accurate diagnosis.

Sano $\mathrm{R}$ et $\mathrm{al}^{19}$ suggested that comparison of angle parameters in supine and prone positions can be another method to predict risk for angle closure even in absence of positive prone provocation test (PPT). In this study while the intraocular pressure was higher after PPT than before the test, every subject was evaluated as negative for PPT. Mean value of every parameter examined was lower in the prone position than in the supine position (AOD 250: 114 microns, vs 128 microns, AOD 500: 121 microns vs 144 microns, TIA: 12.1 degrees vs 15.5 degrees, ACD: 1966 microns vs 2002 microns), and the change in ACD was statistically significant. Thus the anterior chamber configuration of patients with narrow angle is changed in prone position. Such a change can occur even in patients classified as negative for PPT.

Ishikawa $\mathrm{H}$ et $\mathrm{al}^{20}$ added that the more posterior the iris insertion on the ciliary face, the less likely the provocative test will be positive.

Indentation UBM gonioscopy is a very useful method for observing the angle and diagnosis of relative pupillary block, peripheral anterior synechie, and plateau iris configuration. ${ }^{14}$

\section{Dark Room Provocative Test}

Dada et al also performed an evaluation of the effect of the Valsalva maneuver on the anterior chamber configuration using

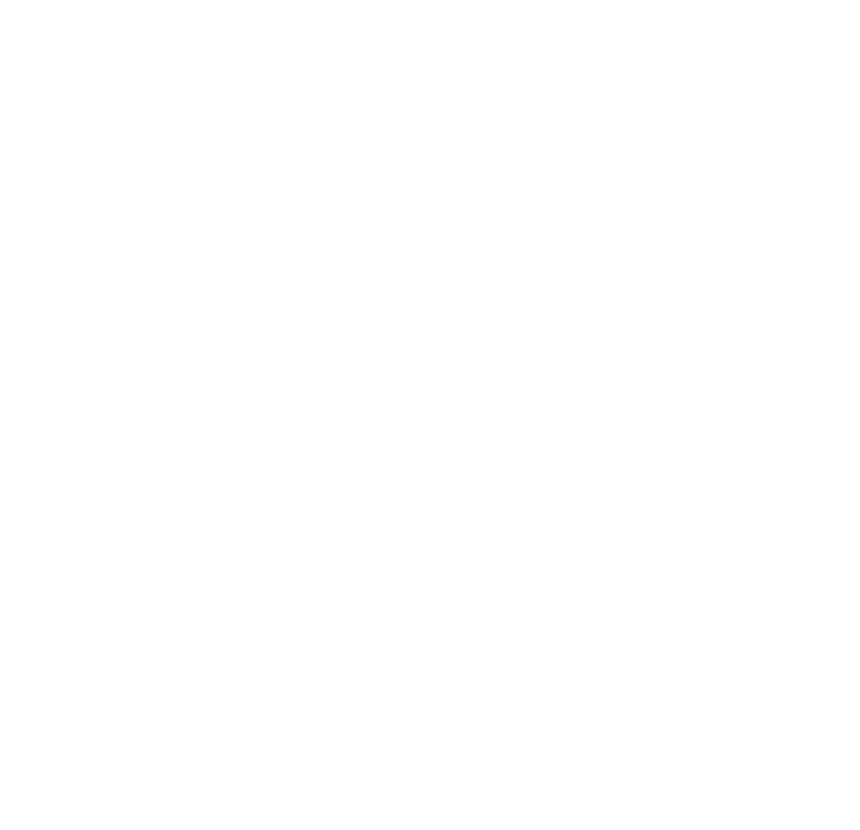

Fig. 6: Before

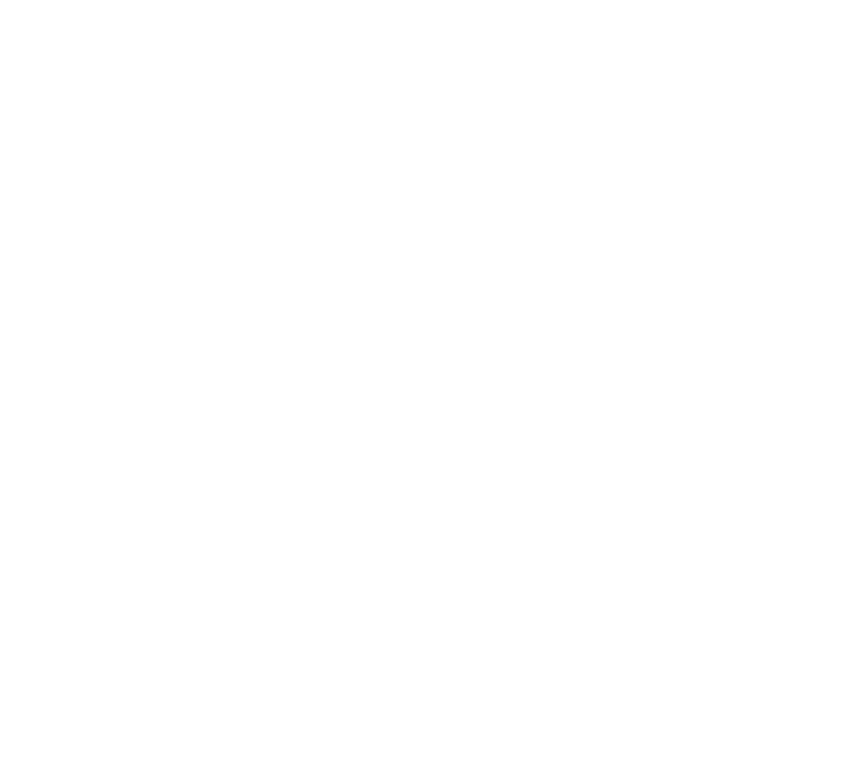

Fig. 7: After

the UBM. Significant elevation of the intraocular pressure, narrowing of the anterior chamber angle recess, thickening of the ciliary body and increase in the iris thickness was seen during the Valsalva maneuver. The study showed that the Valsalva maneuver which comes into play in many activities of daily living, may lead to angle closure in eyes which are anatomically predisposed to primary angle closure glaucoma. ${ }^{21}$

UBM can be used as a dynamic differentiating method to compare the morphologic characters of the anterior chamber 
angle of the high-risk chamber angle. ${ }^{15,22}$ Different illumination leves influence the UBM examination of the anterior chamber angle significantly, and the angle morphologic characters should be checked under different illumination conditions if it seems to be closed.

\section{Congenital Glaucoma}

Wang $\mathrm{N}$ et $\mathrm{al}^{23}$ used UBM to investigate the characteristics of congenital glaucoma. They found that UBM can show the dysplasia of anterior angle, iris, ciliary body and scleral spur of primary congenital glaucoma. The base of iris is thin, the ciliary body is small, and the anatomic characteristic is not clear. The thickness of iris and size of ciliary body of primary congenital glaucoma are significantly smaller than those of normal control group. The dysplasia of iris and ciliary body may play a relative important role in the pathogenic mechanism of congenital glaucoma.

Another study ${ }^{24}$ evaluating the anterior segment changes in primary infantile glaucoma (PIG) suggests different results. According to the study, no matter the severity of the disease or the age at onset, the most significant characteristics of the diseased eyes are relative positional changes between scleral spur and angle apex. In three forth of the eyes, the scleral spur lay in the lateral or posterior-lateral site of the angle apex and in one forth of the eyes the site of the tip of scleral spur was parallel with the iris root insertion. The thickness and length of ciliary processes in the eyes of PIG were greater than that of the normal infantile eyes. The ciliary processes were anteriorly displaced, pulled towards the lens, and part of them got into contact with the iris. The relative positional changes between scleral spur and angle apex in diseased eyes indicate that the poor development of scleral spur and the iris anterior insertion are the basic pathogenesis in PIG.

In cases of cloudy cornea and unknown previous glaucoma surgery, UBM can be used to identify the type and localization of previous surgery in congenital glaucoma, thus assisting surgical planning for subsequent glaucoma management. ${ }^{25-27}$ The correlation between UBM morphology and the effectiveness of filtering surgery is less convincing than previously demonstrated in adults, possibly underlining the importance of individual nonsurgical factors for prognosis in congenital glaucoma.

\section{Determining the Mechanism of Secondary Glaucoma}

In the pigment dispersion syndrome there is a classical picture on the UBM which includes widely opened angle and typical posterior bowing of the peripheral iris ${ }^{6}$ (Fig. 8). MendezHernandez et $\mathrm{al}^{28}$ studied the effect of Yag PI on iris configuration in pigmentary glaucoma. In their study they found that PI rectified the posterior bowing of iris and reduced the drugs required.

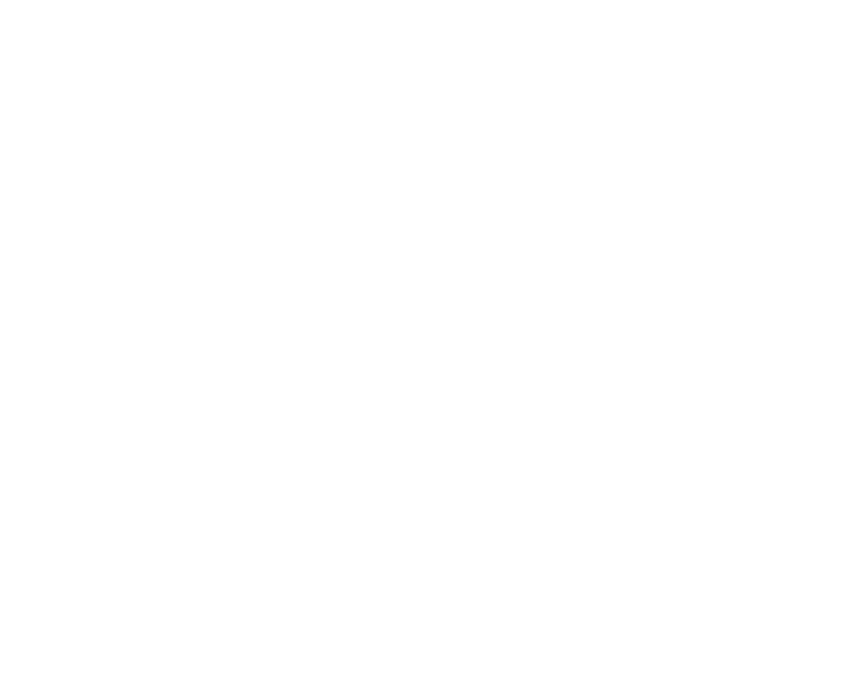

Fig. 8: Pigment dispersion syndrome

Pillunat LE et $\mathrm{al}^{29}$ studied UBM in pigmentary glaucoma and effect of YAG iridectomy on anterior segment structures and IOP. The results show that irido-zonular contact does not exist in every patient with pigmentary glaucoma. Therefore, it seems possible that more than one pathogenic mechanism is involved in pigmentary glaucoma. Only in patients with iridozonular contact, however, laser iridotomy significantly reduces intraocular pressure.

In eyes with peripheral anterior synechiae, UBM can reveal the extent of iridocorneal adhesions, even if the cornea is hazy or opaque (Figs 9 and 10). The UBM has been able to differentiate between primary angle closure and secondary angle closure due to processes such as lens swelling and dislocation, massive hemorrhagic retinal detachment pushing the lens and iris anteriorly, and multiple neuroepithelial cysts of the iridociliary sulcus.

Okamoto $\mathrm{F}$ et $\mathrm{al}^{30}$ demonstrated that there is an iris hypoplasia and a ciliary body hypoplasia in aniridia. Anterior inclination of the ciliary process was also found, which was thought to be at least partly responsible for the shallow anterior chamber (Fig. 11).

In Sturge-Weber syndrome associated glaucoma, UBM can show the presence of dilated intrascleral vessels and supraciliary fluid supporting the hypothesis of increased episcleral venous pressure as the cause of elevated intraocular pressure in this condition. $^{31}$

Zhang $\mathrm{M}$ et al ${ }^{32}$ compared UBM findings of anterior segment between normal subjects and three clinical types of ICE syndrome: progressive iris atrophy (PIA), Chandler's syndrome (CS), and Cogan-Reese syndrome (CRS). UBM was found to be more effective in detecting peripheral anterior synechiae (PAS) and iris atrophy than slit lamp microscopy and gonioscopy, mainly because of corneal edema in patients with CS. Four out 


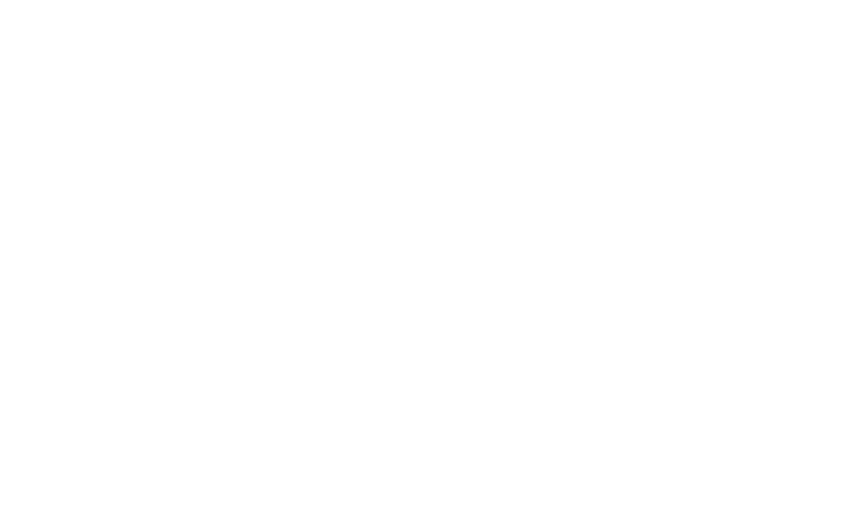

Fig. 9: Peripheral anterior synechiae

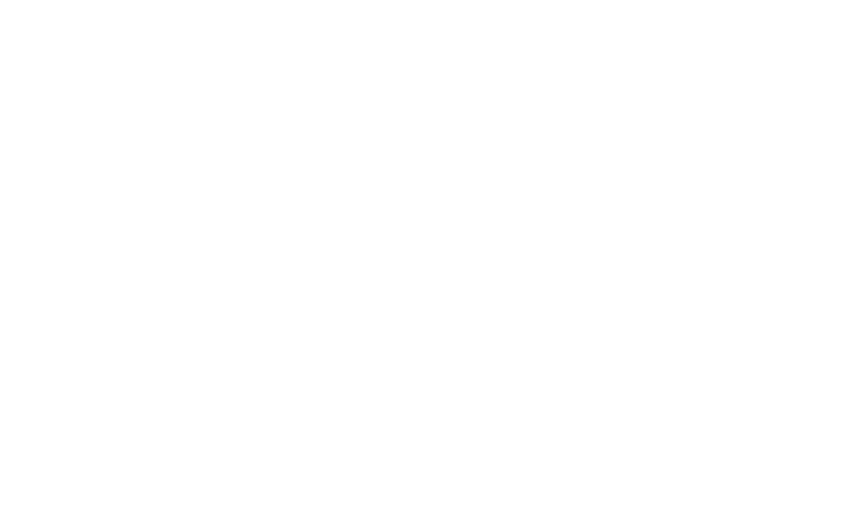

Fig. 10: Iris bombe

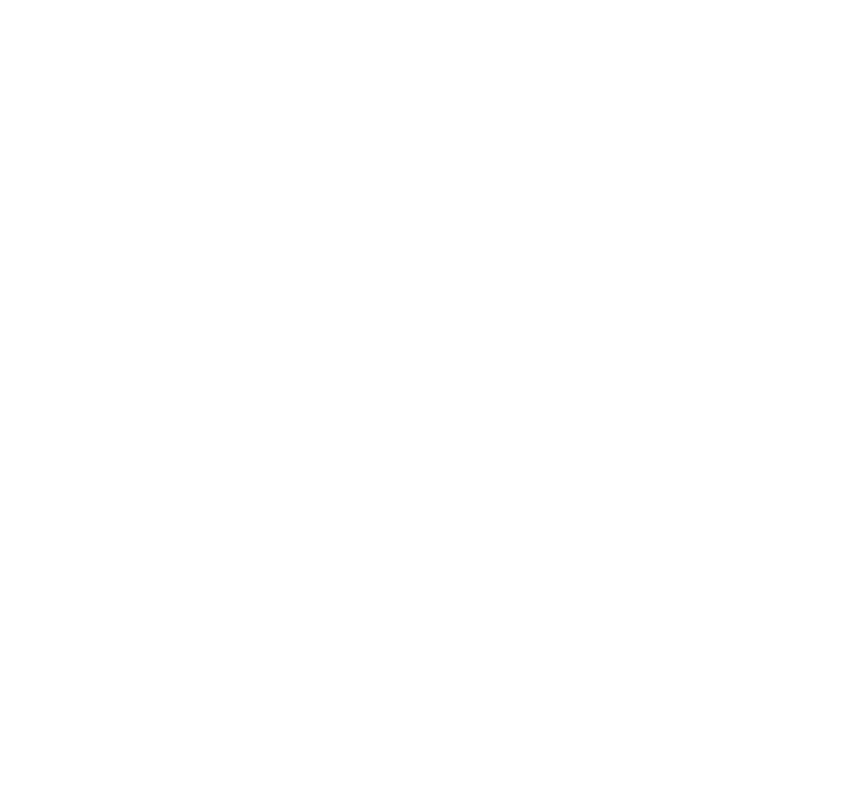

Fig. 11: Aniridia-iris and ciliary hypoplasia

of 11 patients with unilateral ICE syndrome had shallow or closed anterior chamber angles in their fellow eyes. Two of them successfully responded to laser peripheral iridotomy. Thus UBM is an effective method to reveal the anterior segment features and provides a useful tool in the diagnosis of ICE syndrome. Different subtypes of ICE syndrome may have different UBM manifestations. UBM can help to identify angle closure in the fellow eye of unilateral ICE syndromes.

\section{Post-traumatic Glaucoma}

After blunt ocular trauma, UBM can be used to evaluate irisangle abnormalities including angle recession, iridodialysis and cyclodialysis, and to illustrate the presence and extent of blood clots (Figs 12 and 13). Angle recession is characterized on UBM by a posterior displacement of the point of attachment of the iris to the sclera, a widening of the ciliary body face with no disruption of the interface between the sclera and ciliary body. In the acute stage, the post-traumatic recess is usually filled with blood. In contrast, in cyclodialysis, the ciliary body is detached from its normal location at the scleral spur. ${ }^{33,34}$ It is particularly useful in the presence of hazy media, hypotony, and/or abnormal anterior segment anatomy. UBM can also identify occult zonular damage in patients with anterior segment trauma. ${ }^{35}$ The ability to diagnose zonular rupture preoperatively is of significant benefit to the surgeon and might reduce the chance of intraoperative complications.

\section{Pseudophakic and Lens Induced Glaucoma}

The UBM can diagnose various types of lens induced glaucomas such as Phacomorphic glaucoma and glaucoma due to anterior subluxation of lens. It is helpful to know the circumference of intact zonules and the extent of zonular dialysis in pseudoexfoliation syndrome. In case of IOL induced glaucoma, it can clearly delineate the position of the optic and haptic and is especially helpful in pseudophakic bullous keratopathy cases to determine the cause for glaucoma (Figs 14 and 15).

\section{Secondary Glaucoma after Retinal Surgery}

This retrospective study ${ }^{36}$ was undertaken to evaluate the utility of Ultrasound Biomicroscopy (UBM) in the assessment of the anterior chamber in patients affected by silicone oil-related glaucoma after vitreoretinal surgery. UBM showed high reflectivity of angular structure, anterior chamber alterations and a fairly trophic ciliary body.

Wei W et $\mathrm{al}^{37}$ studied UBM changes after buckling surgery. The study shows that the anterior chamber angle becomes narrower, the angle open distance 500 decreases, the trabeculariris angle decreased in degree, the angle of the scleral lateral side and iris long axis decreases, but the depth of the central anterior chamber has no obvious change. Ciliary body detachment and edema are often found after scleral buckling surgery, and postoperatively the central anterior chamber depth 


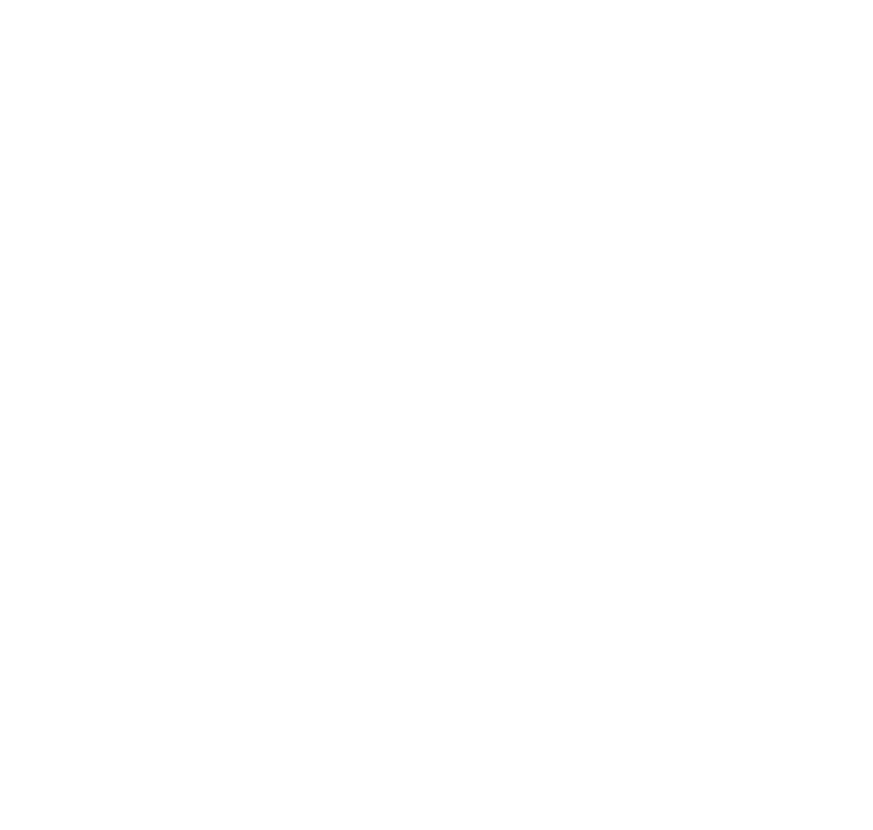

Fig. 12: Angle recession

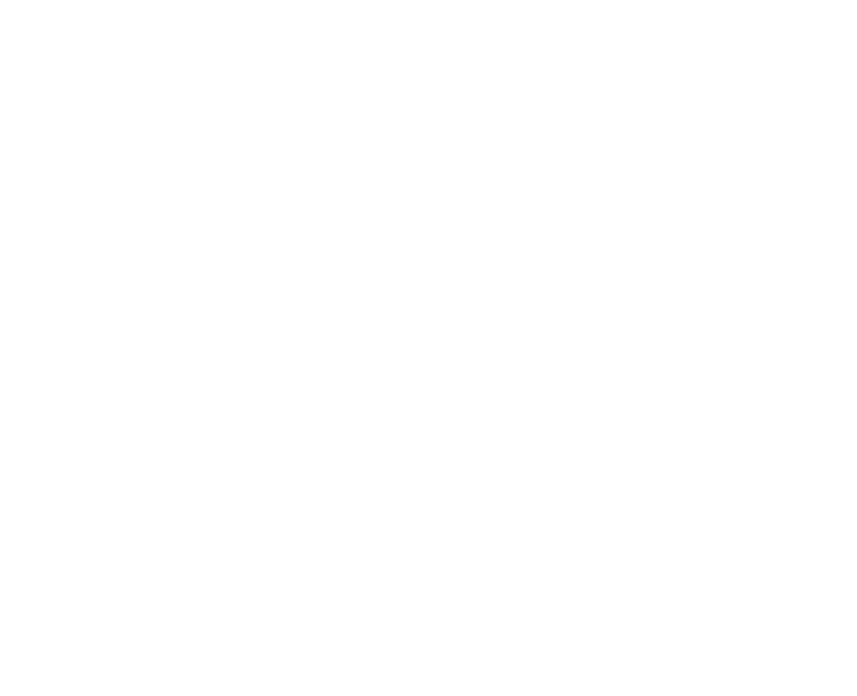

Fig. 13: Iridodialysis

has no obvious change; ciliary body detachment and edema perhaps are related to the angle-closure glaucoma after the surgery.

\section{Diagnosis of Malignant Glaucoma}

Ciliary block or aqueous misdirection presents the greatest diagnostic challenge for the ophthalmologists and the UBM is an important tool in this condition. Imaging shows an extremely shallow anterior chamber, occluded angle, forward rotation of the ciliary body with or without fluid in the suprachoroidal space. ${ }^{6}$

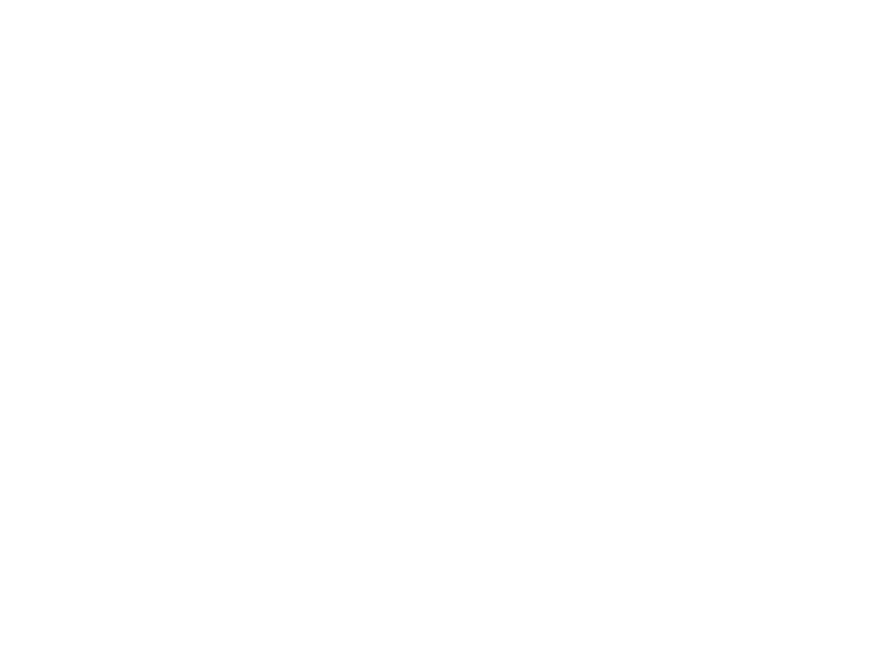

Fig. 14: Secondary angle closure by $\mathrm{ACIOL}$

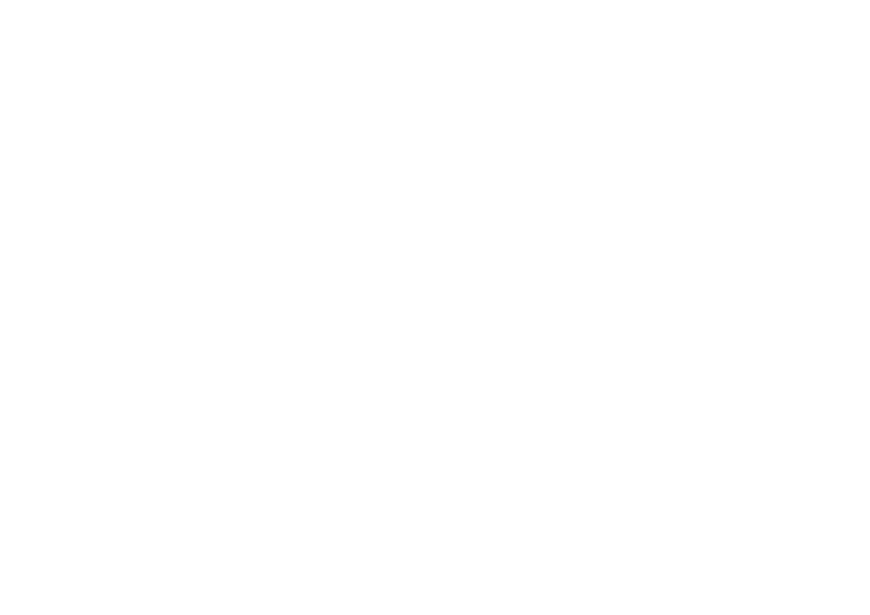

Fig. 15: Intact zonules

Liu L et $\mathrm{al}^{38}$ studied the onset of malignant glaucoma in living eyes. The mechanism of malignant glaucoma is associated with the abnormal relationship among anterior vitreous, ciliary process and lens periphery. Fluid in the supraciliary space makes the ciliary process closer to the lens periphery. It is one of the factors causing cilio-lens block. The ultrasound biomicroscopic image is a new better practicable method to diagnose malignant glaucoma during its onset compared with other methods at present used in the clinical work. It is much more valuable to differentiate the pupillary block glaucoma from malignant glaucoma by using ultrasound biomicroscopy.

Sometimes malignant glaucoma is suspected in cases of secondary glaucoma like iridocyclitis, lens subluxation resulted from trauma and glaucoma filtration surgery. ${ }^{39}$

In the cases of lens subluxation, the main characteristic was the increase of the distance between the lens and the ciliary process at the site of subluxation and the lens forward movement. 
In the cases of iridocyclitis, anterior and posterior synechiae were the main characteristics. In case of glaucoma filtration surgery there were two main characteristics: One was that the distance between the ciliary process and the equator of lens existed obviously, and the other was the occlusion of the inner ostium of glaucoma filtration surgery or peripheral iridectomy. Thus UBM has important value in the diagnosis of secondary pupillary block glaucoma and its differentiation from malignant glaucoma. Whether the posterior chamber exists or not is the differential point between the pupillary block glaucoma and the malignant glaucoma.

\section{Secondary Angle Closure after Opaque Graft}

Secondary angle closure caused by anterior synechiae formation is one of the important causes of Post-PKP Glaucoma (PPKG) in eyes with opaque grafts. UBM serves as a useful tool for anterior-segment evaluation in such cases and can help in planning the site for glaucoma filtering surgeries and drainage devices ${ }^{40}$ (Fig. 16).

\section{Evaluation of Cysts and Tumors Causing Angle Closure}

Cysts and solid tumors of the anterior segment can be imaged in great detail with UBM. ${ }^{41}$ This technology can be used to determine the internal character of a lesion (solid or cystic), to ascertain whether the lesion involves the anterior ciliary body or is restricted to the iris, and to measure the full extent of the lesion. UBM can reveal whether the lesion involves only partial thickness or full thickness of the stroma and can thereby aid in surgical planning. It allows measurement of the lesion's thickness and determination of the presence or absence or intraocular invasion. It also confirms the presence, character, and extent of ciliary body tumors and often reveals the route of access of the tumor to the surface by way of a scleral emissary canal. With the UBM one can follow-up the progression or regression of the tumor with exact documentation of the dimensions of the tumor (Figs 17 to 19).

Bhende $\mathrm{M}$ et $\mathrm{al}^{42}$ used UBM in the identification of an intraocular nematode in a case of suspected nematode-induced uveitis, which was not detected on clinical examination.

\section{Study of Ciliary Body Blood Flow}

With the new UBM machines one can study the blood flow in the ciliary body and see the effect of various medications/ surgery on the ciliary circulation.

Uses of UBM in Laser and Glaucoma Surgery

\section{Determining Patency and Effect of Laser Iridotomy}

After Nd-YAG laser iridotomy for angle closure, UBM can show whether the iridotomy is partial thickness or full thickness and

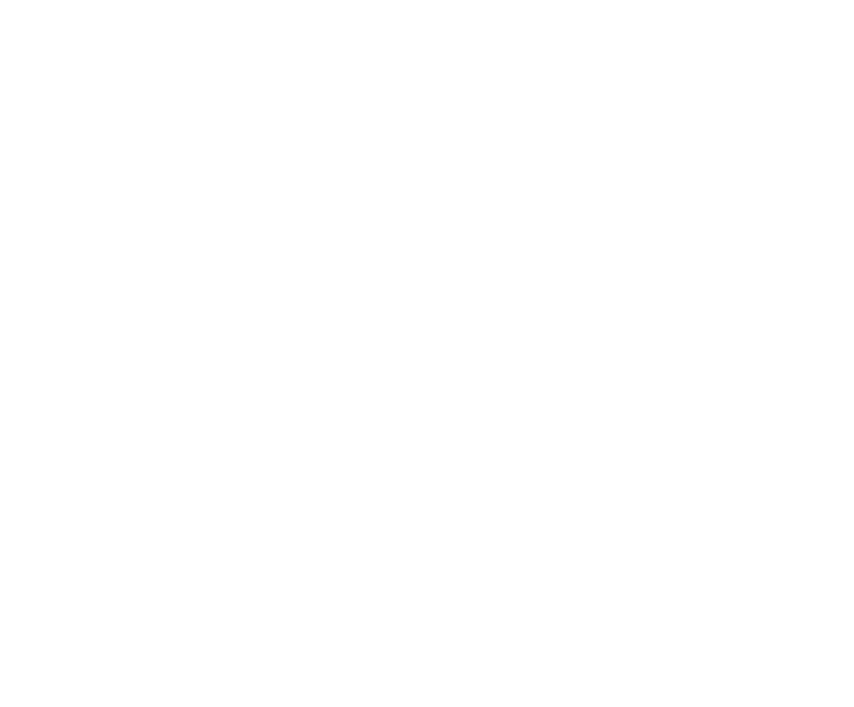

Fig. 16: Extensive synechia in a case of opaque graft

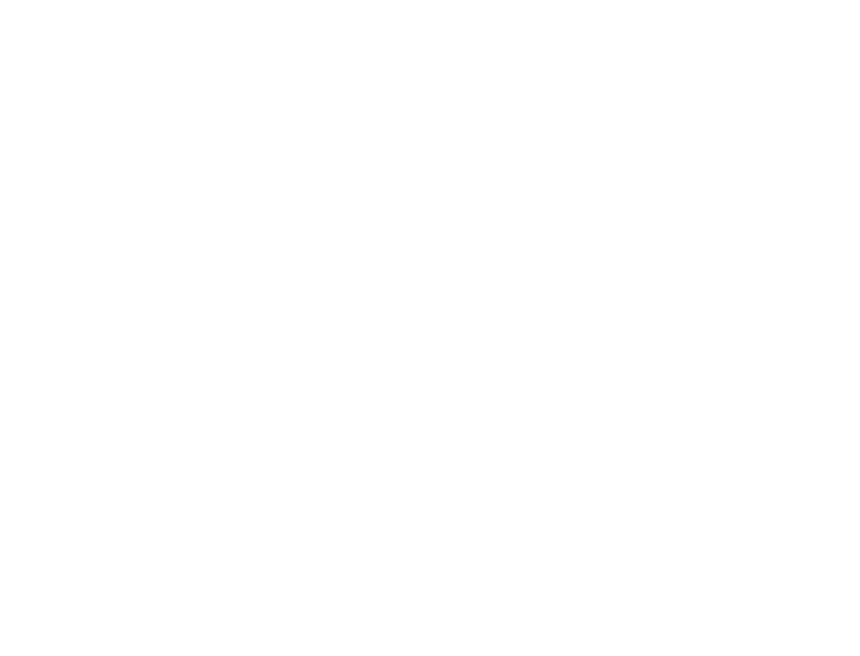

Fig. 17: Iris cyst

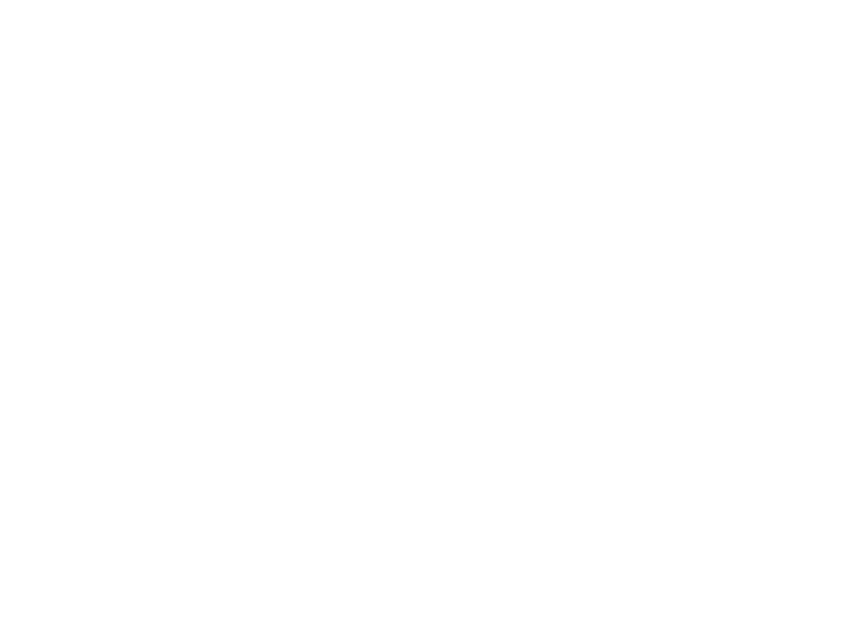

Fig. 18: Ocular surface squamous neoplasia not invading angle 


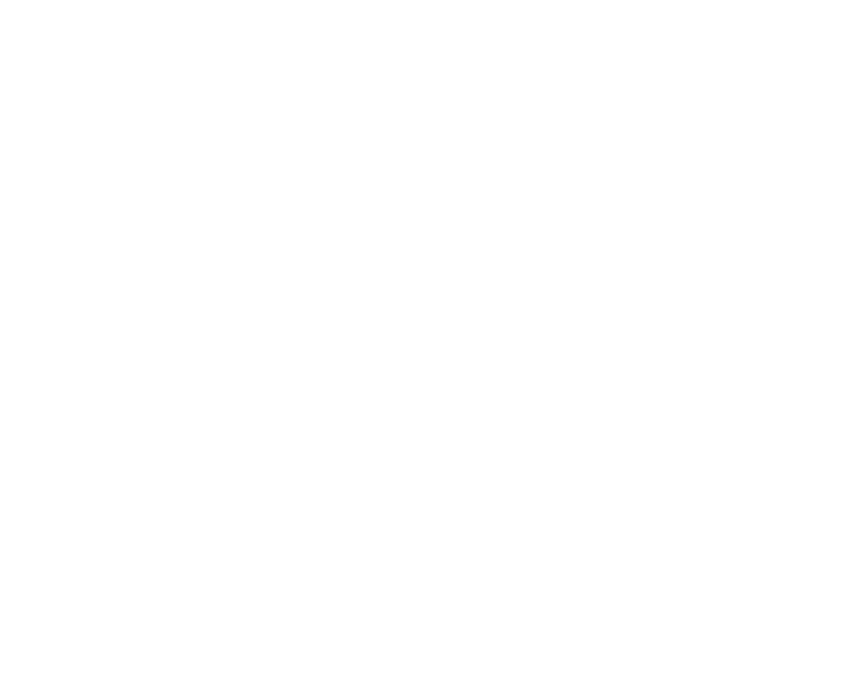

Fig. 19: Ciliary body tumor

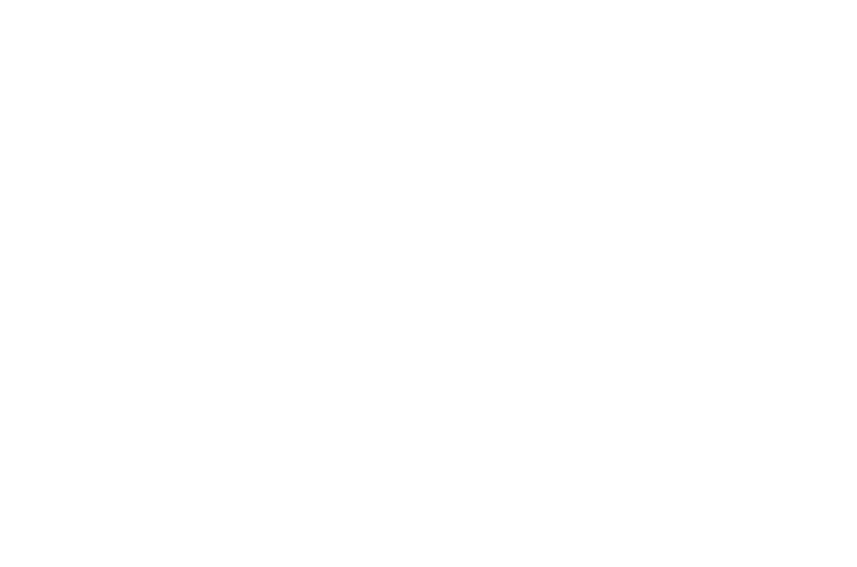

Fig. 20: Nd-YAG iridotomy

whether the plane of curvature of the peripheral iris has changed, compared with the pretreatment findings ${ }^{43}$ (Fig. 20).

In case of pigment dispersion syndrome, laser iridotomy eliminates the pressure gradient between anterior and posterior chamber and relieves reverse pupillary block. The UBM can clearly show the flattened iris after iridotomy. ${ }^{44}$ Any damage to the lens caused by the laser can also be highlighted on the UBM. Subclinical choroidal effusion observed by UBM frequently occurs after LPI (laser peripheral iridotomy). Ciliochoroidal effusion appeared more often in the Ar-LPI group (10 eyes, 52\%) than in the Ar-YAG-LPI group. ${ }^{45}$

Dada et $\mathrm{al}^{46}$ studied changes in anterior segment morphology after LPI in primary angle closure (PAC) and primary angle closure glaucoma (PACG) using ultrasound biomicroscopy (UBM). They found LPI leads to a widening of the anterior chamber angle and a deepening of the anterior chamber in eyes with PAC. It does not significantly change any anterior segment parameters in eyes with PACG. Kaushik et $\mathrm{al}^{47}$ added that LPI significantly widened the anterior chamber angle in the quadrant with LPI and the quadrant furthest away in patients of CACG with established glaucomatous damage.

Residual angle closure after iridotomy is common, especially in eyes with primary angle closure and poorly controlled IOP or glaucomatous optic neuropathy. Nonaka $\mathrm{A}$ et $\mathrm{al}^{48}$ studied residual angle closure after iridotomy and found in $38.6 \%$ of eyes; this was confirmed functionally by the dark room prone position test and morphologically by UBM. They found that cataract surgery was effective to resolve completely the residual angle closure after iridotomy and lower IOP.

\section{Determining Functional Status of a Filtering Surgery}

After trabeculectomy UBM can show whether the sclerostomy aperture is patent or blocked internally, whether the peripheral iridectomy is patent and whether the filtering bleb is flat, shallow, or deep (Figs 21 and 22).

After glaucoma drainage device surgery, UBM can shows the position of the tip of the tube and whether its orifice is open or plugged.

The grading of the bleb is done according to intrableb reflectivity, visibility of route under the scleral flap, formation of cavernous fluid filled space, and bleb height. Following four grades have been described:

- Low reflective L

- High reflective $\mathrm{H}$

- Encapsulated E

- Flat F

Eyes with good IOP control mainly have 'L' type blebs, these have low to moderate intrableb reflectivity, visible intrascleral route and higher intrableb height. Flat and encapsulated blebs generally denote a surgical failure.

Avitabile $\mathrm{T}$ et $\mathrm{al}^{49}$ studied correlation of bleb morphology on UBM and functional status with effect of laser suture lysis.

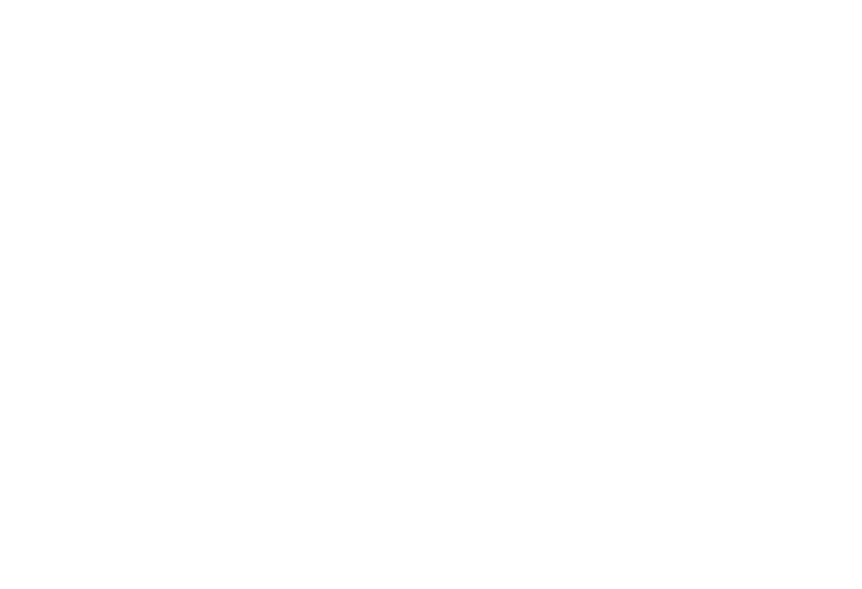

Fig. 21: Trabeculectomy 


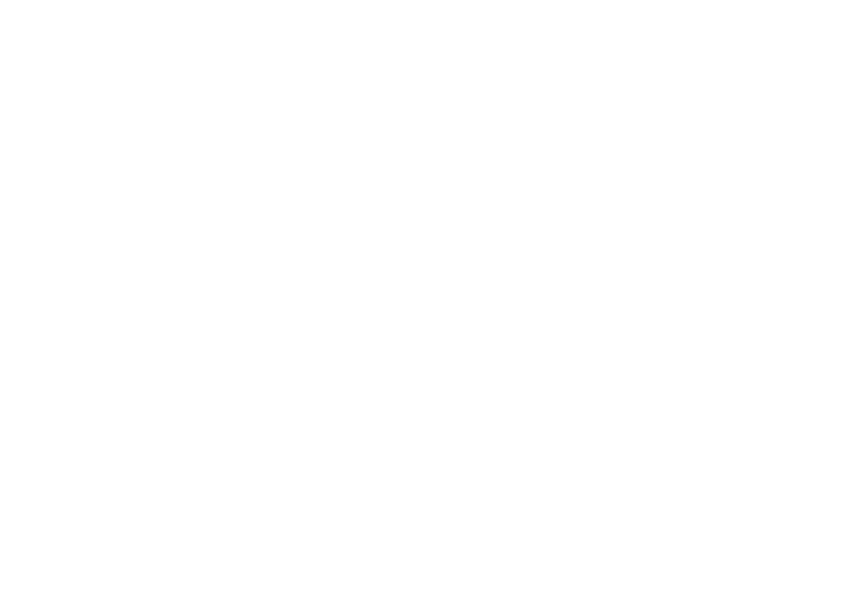

Fig. 22: Filtering bleb

They found a statistically significant correlation between the UBM classification of function and the IOP control level. Both well-functioning and failed trabeculectomies could be identified by UBM. The UBM images of eyes with good IOP control are characterized by better visibility of the route under the scleral flap and a low reflectivity inside the bleb. Thus UBM can be a useful method to study and explain the mechanisms of filtering structures and, together with IOP control, to evaluate the bleb function.

\section{Nonpenetrating Deep Sclerectomy}

UBM may be used in eyes which have undergone nonpenetrating-deep sclerectomy (NPDS) to evaluate the functional status of the surgery. It can evaluate the thickness and demonstrate a nonperforated continuous trabeculo-descemets membrane. In patients undergoing deep sclerectomy, UBM examination after long-term follow-up shows the presence of an intrascleral space and a filtering bleb. ${ }^{50}$ Collagen implants used to augment deep sclerectomy can also be visualized using this technology. A grading of bleb similar to the trabeculectomy bleb has also been used for NPDS blebs. Contreras et al studied the new non-absorbable implant and proved to be effective in POAG cases. ${ }^{51}$ Information provided by UBM is useful and assists in understanding the mechanism of action of deep sclerectomy.

Wang Y et $\mathrm{a}^{52}$ studied causes of failure of NPDS with SKGeL (a hyaluronic acid biological gel) implant and possible treatment option. The examination of UBM showed that the filtering bleb disappeared and there was a liquid chamber under the superficial scleral flap in every failure case. The scarring at conjunctivaTenon's capsule-superficial scleral flap interface was the most important cause of NPTS with SKGeL implant failure. A repeated surgery with MMC through the initial surgical site may be a choice for the failure cases.

Park $\mathrm{M}$ et $\mathrm{al}^{53}$ studied Ultrasound biomicroscopy of intrascleral lake after viscocanalostomy and cataract surgery.
They found combined viscocanalostomy and cataract surgery lowered IOP without bleb formation. Postoperatively, the size of the lake and IOP decreased, suggesting parallel reduction of the two. The lake was undetected ultrasonographically in one third of the cases 1 year postoperatively.

\section{Other Surgeries}

Ultrasound biomicroscopy of the anterior chamber angle demonstrates restoration of an open anterior chamber angle after goniosynechialysis. ${ }^{54}$ Experimental study on UBM-guided chamber angle surgery was done by Dietlein TS et al. ${ }^{55}$ Mechanical goniopuncture or punctual Er:YAG laser trabecular ablation was performed without operating microscope or gonioscopy, but with real-life ultrasound biomicroscopy monitoring with a $50 \mathrm{MHz}$ transducer. It was observed that the instruments could be clearly visualized within the chamber angle and disturbing artifacts were only minimal when using mechanically fixed instruments in slow motion. Topographic localization, tissue contact, and penetration depth of the instruments entering the scleral were well illustrated as far as the technical resolution limits of UBM would allow. UBM-guided surgery may be considered for clinical use in humans in future. UBM is instrumental in diagnosing the presence and cause of occlusion of aqueous drainage tubes ${ }^{56}$ (Fig. 23). In eyes with Post Penetrating Keratoplasty glaucoma, the UBM is helpful in identifying the degree of irido-cornea apposition, measuring the corneal thickness, evaluating the lens/IOL and establishing the cause for raised IOP. It also aids the corneal surgeon in planning regrafting in such cases.

Pereira FA et al $^{57}$ studied effect of cataract surgery on angle structures. After phacoemulsification and foldable IOL implantation, UBM revealed that the iris diaphragm shifted backward, deepening the anterior chamber by approximately 850 microm and widening its angle by approximately 10 degrees. These findings may be of clinical significance in eyes with angleclosure glaucoma or with occludable angles.

\section{Evaluation of Postoperative Complications after Trabeculectomy}

After any type of glaucoma filtering surgery, UBM can be used to detect and evaluate the extent of postoperative complications such as ciliochoroidal effusion and cyclodialysis (Fig. 24). In ciliochoroidal effusion UBM shows the ciliary body to be edematous and separated from the sclera by a sonolucent collection of supraciliary fluid. Many ciliochoroidal effusions that are too limited in extent to be detectable by indirect ophthalmoscopy and slit lamp biomicroscopy can be imaged by UBM. Study done by Sugimoto $\mathrm{K}$ et $\mathrm{al}^{58}$ revealed that suprachoroidal fluid was present more frequently at an early stage after trabeculectomy and results indicate that the presence of SCF is related to the early low IOP after trabeculectomy, while that the disappearance of SCF induces the elevation of IOP. 


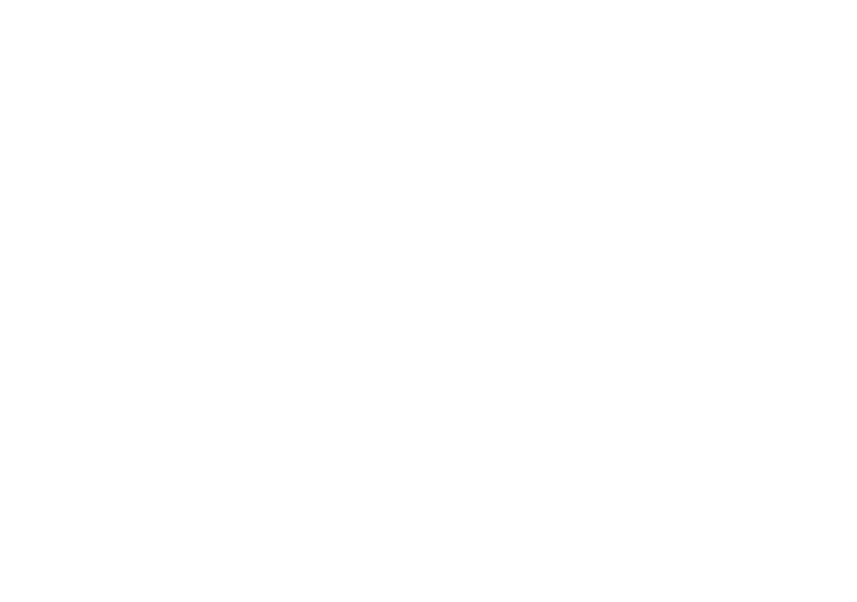

Fig. 23: Aqueous drainage device

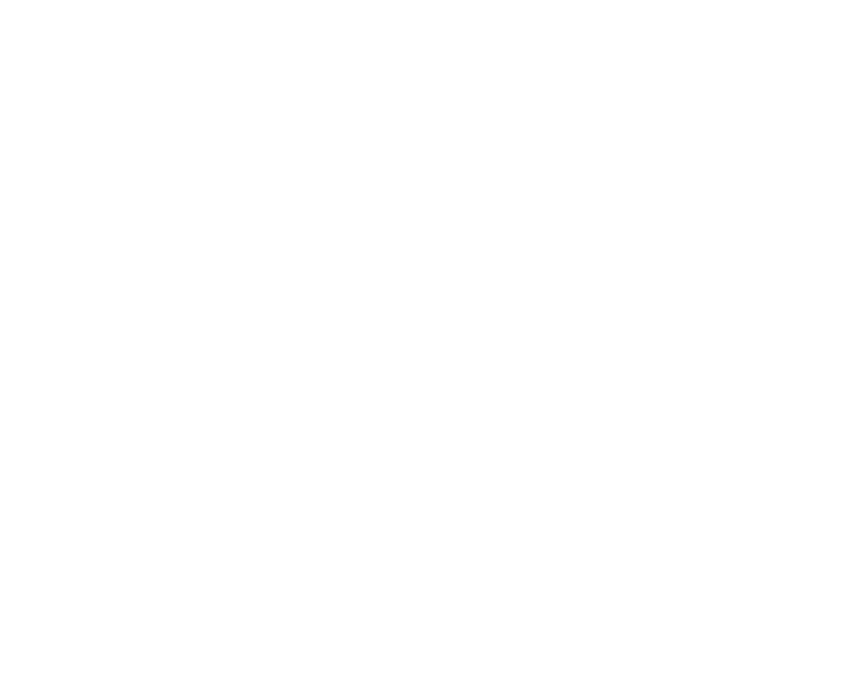

Fig. 24: Choroidal effusion

Information about changes in the trabeculectomy ostium and adjacent structures related to flattening of the anterior chamber in the early postoperative period may be important for management.

Grigera D et al ${ }^{59}$ studied the pathophysiology of flat anterior chamber without bleb leak. Ring-shaped effusions were detected on UBM even in cases in which conventional ultrasonography showed no positive results. UBM is a helpful tool in the diagnosis and management of flattening of the anterior chamber after trabeculectomy. In cyclodialysis UBM shows a well-defined separation between the uveal tissue and the sclera in the region of the scleral spur.

\section{QUANTITATIVE ULTRASOUND BIOMICROSCOPY}

The UBM measurement software calculates distance and area by counting the pixel numbers along the measured line or within the measured area and multiplies the pixel counts by the size of pixel. The UBM provides approximately $25 \mu \mathrm{m}$ of axial and 50 $\mu \mathrm{m}$ of lateral resolution.

The following parameters are used for doing an objective analysis of the anterior chamber angle structures, with the scleral spur taken as the reference point ${ }^{1}$ (Figs 25 to 27).

1. The trabecular-iris angle (TIA) is measured with its apex at the iris recess and the arms of the angle passing through a point on the trabecular meshwork at $500 \mu \mathrm{m}$ from the scleral spur and the point on the iris perpendicularly opposite.

2. The angle opening distance $250 / 500$ (AOD 250/AOD 500) is the distance between the posterior corneal surface and the anterior iris surface measured on a line perpendicular to the trabecular meshwork, 250/500 $\mu \mathrm{m}$ from the scleral spur.

3. The trabecular-ciliary process distance (TCPD), is measured on a line extending from the corneal endothelium at $500 \mu \mathrm{m}$ from the scleral spur perpendicularly through the iris, to the ciliary processes.

4. The iris thickness 1 (ID 1), is the iris thickness measured along the same line as the TCPD. ID2 is the iris thickness at $2 \mathrm{~mm}$ from iris root and ID3 is maximum iris thickness near pupillary margin.

5. The iris-ciliary process distance (ICPD), is the distance measured from the posterior iris surface (iris pigmented epithelium to the ciliary process along the same line as the TCPD.

6. The iris-lens contact distance (ILCD), is measured along the iris pigmented epithelium from the pupillary border to the point where the anterior lens surface leaves the iris.

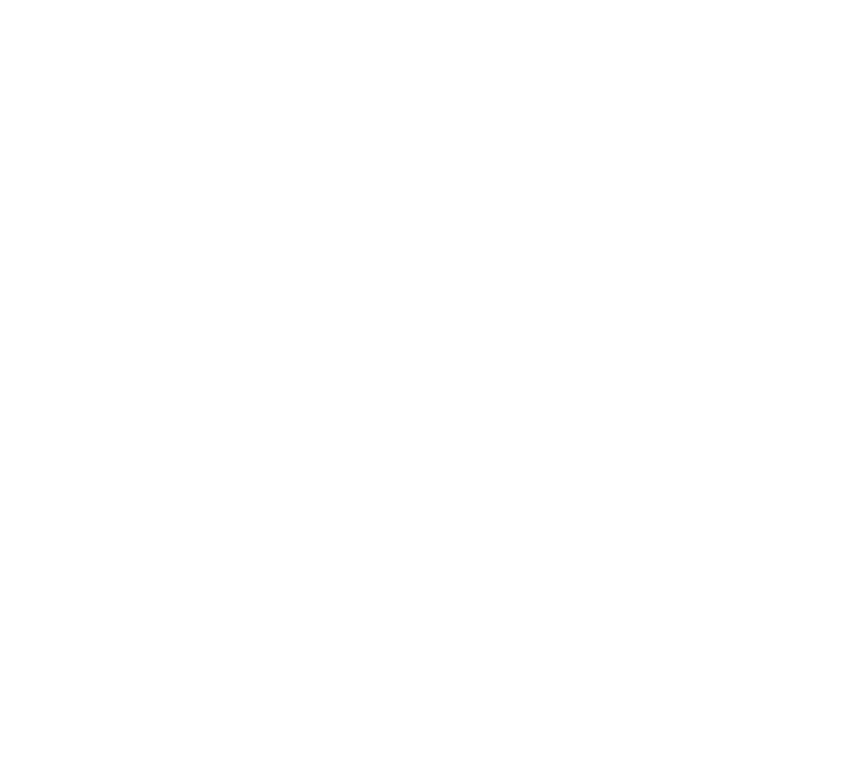

Fig. 25: Angle parameters 


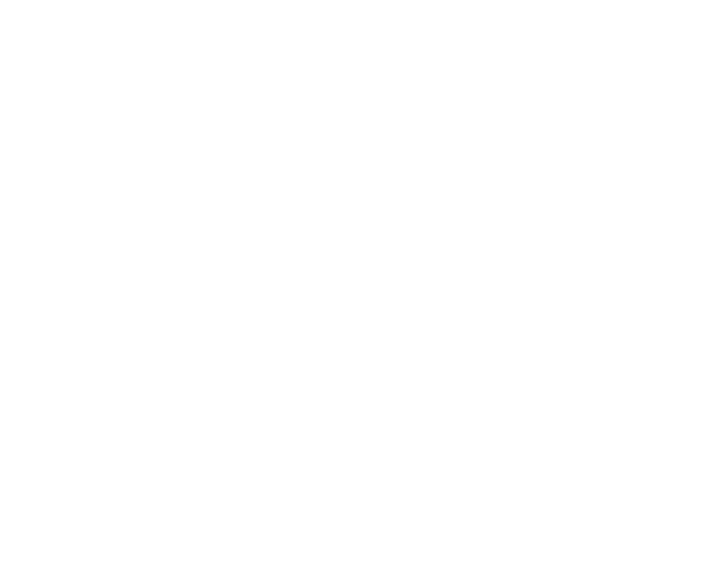

Fig. 26: Measurement of angle parameters

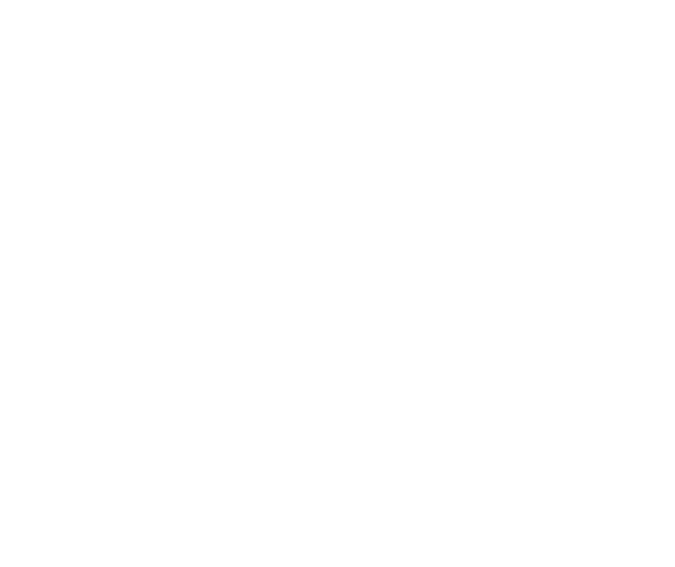

Fig. 27: Angle recess area

\section{CLINICAL APPLICATIONS OF QUANTITATIVE ULTRASOUND BIOMICROSCOPY}

Quantitative parameters have been used in differentiating angle closure and normal eyes ${ }^{6}$ and to show the iris convexity related to age. ${ }^{60}$

\section{QUANTIFICATION OF THE ANTERIOR CHAMBER ANGLE}

With the UBM one can draw calipers and directly measure the angle recess precisely. This is a very objective method which is not possible with gonioscopy. It helps to determine the exact degree of angle closure and assess whether a patient is predisposed to angle closure. An automated analysis of parameters can be performed with the UBM Pro software (Fig. 28).

Ishikawa $\mathrm{H}$ et $\mathrm{al}^{61}$ described a quantitative method for measuring the iridocorneal angle recess area, and, using this, to

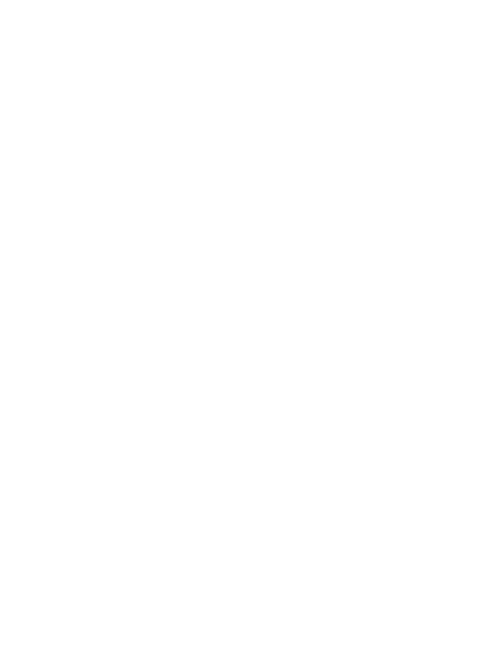

Measurement Type

C. Distance

6. Angle

$C$ Aves

Resul: 88 (degee)

Instruction

LEFT mouse bution

Cock an inspe 3 tmes to mearue angle slating poink clpping point, and ending point

RIGHT mouse button

Togos ENLARGE mode crVoth

Fig. 28: Quantification of the angle

evaluate factors associated with appositional angle-closure during dark room provocative testing using ultrasound biomicroscopy (UBM). They calculated an ARA linear regression formula which provides useful quantitative information about angle recess anatomy. They concluded that the more posterior the iris insertion on the ciliary face, the less likely the provocative test will be positive.

A study on Indian eyes by Kaushik et $\mathrm{al}^{62}$ put forth the objective measurements of the angle in open and closed angles, and there correlation with clinical grading. They found the mean AOD 250, AOD 500 and TCPD in narrow angles were 58+/- 49 micro, $102+/-84$ micro and $653+/-124$ respectively, while it was 176+/- 47 micro, 291+/- 62 micro and 883+/-94 micro in eyes with open angles $(\mathrm{P}<0.001)$ respectively. The angle width estimated by gonioscopy correlated significantly with the angle dimensions measured by UBM. But UBM measurements are definitely more reproducible. Spaeth GL et al ${ }^{63}$ Studied inter and intraobserver variability in reading UBM and found that the agreement within the same observer and between observers in evaluating the ACA configuration by UBM was excellent.

Another method of quantitative measurement of angle is anterior segment OCT. It was compared with UBM by Radhakrishnan S et al. ${ }^{64}$ The AC angle parameters measured by both OCT and UBM had similar mean values, reproducibility, and sensitivity-specificity profiles. Both OCT and UBM showed excellent performance in identifying eyes with narrow angles.

In an another study Dada et $\mathrm{al}^{65}$ compared the anterior segment parameters using quantitative imaging by ASOCT and UBM. Central corneal thickness, anterior chamber depth and the peripheral irido-corneal angles (temporal and nasal) were assessed and compared. They found an excellent correlation between ASOCT and UBM measurements. There was no significant difference between the mean values of all the anterior chamber parameters. 


\section{EFFECTS OF DRUGS}

UBM has been used to evaluate the effects of drug on anterior chamber angle, iris and ciliary body. It has been found that angle opening is increased after pilocarpine installation in eyes with narrow angle whereas angle opening is decreased in eyes with a wider or normal angle. ${ }^{66}$

Over the past few years the rat has gained prominence as an animal model for the study of glaucoma. Nissirios $\mathrm{N}$ et al ${ }^{67}$ investigated the normal rat anterior segment anatomy in vivo using ultrasound biomicroscopy (UBM) and determined the effect of both cholinergic and anticholinergic medications on angle structures. Although both pilocarpine and cyclopentolate induced angle narrowing, inspection of the ultrasonic images revealed a differential effect. Pilocarpine caused a "pupillary block-like” picture, while cyclopentolate caused crowding of the iris base in the angle.

Marchini $\mathrm{G}$ et al ${ }^{68}$ assessed the effects of $0.005 \%$ latanoprost on the anterior segment geometry and ciliary body thickness using ultrasound biomicroscopy.

The increase of ciliary body thickness, which was measured in vivo by ultrasound biomicroscopy and associated with the intraocular pressure-lowering effect, indirectly supports the mechanism of uveoscleral outflow enhancement induced by latanoprost. These data are in agreement with the biochemical hypothesis of the passage of the aqueous flow through the extracellular spaces of the ciliary muscle.

\section{EFFECTS OF SURGERY}

To evaluate morphological changes after anterior segment surgery, the UBM is a useful tool. It has been shown that endothelial cell loss after laser iridotomy is inversely proportional to the distance of the iridotomy from the endothelium and scleral spur. ${ }^{69}$

After deep sclerectomy, the evaluation of the height and length of collagen implant can be done on the UBM. It has been shown on UBM that these collagen implants dissolve slowly leaving an intrascleral lake. The usual dissolution time for these implants is between 6-9 months. ${ }^{70}$

Aptel et al found UBM and OCT examinations to be useful methods to evaluate outflow mechanisms after deep sclerectomy with Ologen implantation. $^{71}$

Mansouri et al reported that UBM can be used to quantify changes in anterior segment morphology after Nd:YAG laser iridotomy in primary angle closure and PACG in European eyes. They concluded that dimensions of the anterior chamber angle can be significantly influenced by Nd:YAG laser iridotomy in narrow angle European eyes and that UBM examination is a viable tool for the quantitative evaluation of the anterior chamber angle before and after laser iridotomy. ${ }^{72}$

The UBM may find use in ultrasound guided interventions in the anterior segment (e.g. to open up a failed filter) although much work needs to be done on therapeutic uses of this technology.

\section{COMPARISON WITH ANTERIOR SEGMENT OCT}

UBM and anterior segment OCT can both be used in evaluation of angle in glaucoma. Advantages of OCT include non contact technique, easy to learn, can be used to assess refractive surgeries on cornea. Advantages of UBM include ability to visualize ciliary body, pars plana and zonules. Dada et al ${ }^{65}$ compared anterior segment parameters using quantitative imaging by anterior segment OCT and UBM and found comparable results. Radhakrishnan $\mathrm{S}$ et al ${ }^{64}$ also found similar results. Hence both the modalities are useful in aid to glaucoma evaluation with few advantages and disadvantages of each modality.

$\mathrm{Wu}$ et al evaluated the morphology and the function of subconjunctival filtering bleb in patients with glaucoma by slitlamp adapted optical coherence tomography (SL-OCT) and ultrasound biomicroscopy. They reported that SL-OCT scanning has more sensitivity and specificity than UBM in evaluating the function of filtering bleb. The close relationship between the function and the morphological classification provides an important objective basis in evaluating the outcome of antiglaucomatous surgery. ${ }^{73}$

Zhang at al also reported that SL-OCT has greater sensitivity and specificity than UBM in evaluating filtering bleb function and that the morphological classification supported the assessment of bleb function and could provide objective data for evaluating the outcome of antiglaucoma surgery or the need for a second procedure. ${ }^{74}$

Mansouri et al compared ultrasound biomicroscopy and anterior segment optical coherence tomography for evaluation of anterior chamber dimensions in European eyes with primary angle closure and found that AS-OCT measurements are significantly correlated with UBM measurements but show poor agreement with each other. They were of the opinion that ASOCT cannot replace UBM for the quantitative assessment of the AC angle. ${ }^{75}$

\section{CONCLUSION}

Ultrasound biomicroscopy has revolutionized the evaluation of the anterior segment of the eye. The structures surrounding the posterior chamber which were difficult to examine clinically 
now are being imaged and assessed in detail. The qualitative and quantitative evaluation using this technology has contributed to our understanding of the pathophysiology of angle-closure glaucoma, pigmentary glaucoma, secondary glaucoma and a variety of other anterior segment disorders. The use of this technology gives an excellent view of the pathology occurring in the anterior and posterior chambers of the eye and allows objective documentation of the anterior chamber angle and the ciliary body, thereby providing a clear insight into the cause for aqueous obstruction. It also aids in prognostication of a glaucoma case and in establishing the cause for failure of filtering surgery.

\section{REFERENCES}

1. Pavlin CJ, Harasiewicz K, Foster FS. Ultrasound Biomicroscopy of Anterior Segment Structures in Normal and Glaucomatous Eyes. Am J Ophthalmol 1992;113:381-89.

2. Pavlin CJ, Sherar BA, Foster FS. Subsurface Ultrasound Microscopic Imaging of the Intact Eye. Ophthalmology 1990;97:244-50.

3. Pavlin CJ, Harasiewicz K, Sherar BA, Foster FS. Clinical Use of Ultrasound Biomicroscopy. Ophthalmology 1991;98:287-95.

4. Kapetansky FM. A new water bath for ultrasonic biomicroscopy. Ophthalmic Surg Lasers 1997;28:605-06.

5. Esaki K, Ishikawa H, Leibmann JM, et al. A technique for performing ultrasound biomicroscopy in the sitting and prone positions. Ophthalmic Surg Lasers 2000;31:166-69.

6. Ishikawa H, Schuman JS. Anterior segment imaging: Ultrasound biomiscroscopy. Ophthalmol Clin N Am 2004;17:7-20.

7. Marchini G, Pagliarusco A, Toscano A, et al. Ultrasound biomicroscopic and conventional ultrasonographic study of ocular dimentions in primary angle-closure glaucoma. Ophthalmology 1998;105:2091-98.

8. Lowe RF. Causes of shallow anterior chamber in primary angleclosure glaucoma. Am J Ophthalmol 1969;67:87-93.

9. Sihota R, Dada T, Gupta R, et al. Ultrasound Biomicroscopy in the Subtypes of Primary Angle Closure Glaucoma. J Glaucoma. 2005;14:387-91.

10. Nonaka A, Iwawaki T, Kikuchi M, et al. Quantitative evaluation of iris convexity in primary angle closure. Am J Ophthalmol. 2007;143:695-97.

11. Wand M, Grant WM, Simmons RJ, Hutchinson BT. Plateau iris syndrome. Trans Am Acad Ophthalmol Otolaryngol 1997;83:122-30.

12. Kumar, et al. Prevalence of plateau iris in primary angle closure suspects, a UBM study. Ophthalmology 2008;115:430-34.

13. Chiou AG, Mermoud A, Underdahl JP, et al. An Ultrasound biomicroscopic study of eyes after deep sclerectomy with collagen implant Ophthalmology 1998;105:746-50.

14. Matsunga K, Ito K, Esaki K, et al. Evaluation and comparison of indentation ultrasound biomicroscopy gonioscopy in relative pupillary block, peripheral anterior synechia, and plateau iris configuration. J Glaucoma 2004;13:516-19.

15. Garudadri CS, Chelerkar V, Nutheti R. An ultrasound biomicroscopic study of the anterior segment in Indian eyes with primary angle-closure glaucoma. J Glaucoma 2002;11:502-07.
16. Crowston JG, Medeiros FA, Mosaed S, Weinreb RN. Argon laser iridoplasty in the treatment of plateau-like iris configuration as result of numerous ciliary body cysts. Am J Ophthalmol 2005;139:381-83.

17. Argon laser iridoplasty in the treatment of angle closure glaucoma with plateau iris syndrome. Ouazzani BT, Berkani M, Ecoffet M, Lachkar Y. J Fr Ophtalmol 2006;29:625-28.

18. Wang N, Lai M, Cheng X, Ye T. Ultrasound biomicroscopic dark room provocative test. Zhonghua Yan Ke Za Zhi 1998;34:183-86, 12.

19. Sano R, Kurokawa T, Kurimoto Y, et al. Comparison between the anterior chamber configuration in the supine position and that in the prone position in patients with narrow angle. Nippon Ganka Gakkai Zasshi 2001;105:388-93.

20. Ishikawa H, Esaki K, Leibmann JM, et al. Ultrasound biomicroscopy dark room provocative testing: A quantitative method for estimating anterior chamber angle width. Jpn J Ophthalmol 1999;43:526-34.

21. Dada T, Gupta V, et al. Narrowing of the anterior chamber angle during Valsalva maneuver: A possible mechanism for angle closure. Euro J Ophtalmol 2006;16:81-91.

22. Lowe RF. Causes of shallow anterior chamber in primary angleclosure glaucoma. Am J Ophthalmol 1969;67:87-93.

23. Avitabile T, Russo V, Uva MG, Marino A, Castiglione F, Reibaldi A. Ultrasound-biomicroscopic evaluation of filtering blebs after laser suture lysis trabeculectomy.Ophthalmologica. 1998; 212 Suppl 1:17-21.

24. Zhu X, Li Z, Lin D, Tang X, Yang W, Hu S, Wang L. A study of anterior segment structures in primary infantile glaucoma eyes by ultrasound biomicroscopy Zhonghua Yan Ke Za Zhi 1999;35:300-04.

25. Dietlein TS, Engels BF, Jacobi PC, Krieglstein GK. Ultrasound biomicroscopic patterns after glaucoma surgery in congenital glaucoma. Ophthalmology 2000;107:1200-05.

26. Engels BF, Dietlein TS, Jacobi PC, Krieglstein GK. Ultrasound biomicroscopy diagnosis of congenital glaucoma. Klin Monatsbl Augenheilkd 1999;215:338-41.

27. Ultrasound biomicroscopy in infantile glaucoma. Azuara-Blanco A, Spaeth GL, Araujo SV, Augsburger JJ, Katz LJ, Calhoun JH, Wilson RP. Ophthalmology 1997;104:1116-19.

28. Mendez-Hernandez C, Garcia-Feijoo J, Cuina-Sardina R, et al. Ultrasound biomicroscopy in pigmentary glaucoma. Arch Soc Esp Oftalmol 2003;78:137-42.

29. Pillunat LE, Bohm A, Fuisting B, et al. Ultrasound biomicroscopy in pigmentary glaucoma. Ophthalmologe 2000;97:268-71.

30. Okamoto F, Nakano S, Okamoto C, et al. Ultrasound biomicroscopic findings in aniridia. Am J Ophthalmol 2004;137:858-62.

31. Kranemann CF, Pavlin CJ, Trope GE. Ultrasound biomicroscopy in Sturge-Weber-associated glaucoma. Am J Ophthalmol 1998;125:119-21.

32. Zhang M, Chen J, Liang L, Laties AM, Liu Z.Ultrasound biomicroscopy of Chinese eyes with iridocorneal endothelial syndrome. Br J Ophthalmol 2006;90:64-69.

33. Berinstein DM, Gentile RC, Sidoti PA, et al. Ultrasound biomicroscopy in anterior ocular trauma. Ophthalmic Surg Lasers 1997;28:201-07. 
34. Park M, Kondo T. Ultrasound biomicroscopic findings in a case of cyclodialysis. Ophthalmologica 1998;212:194-97.

35. McWhae JA, Crichton AC, Rinke M. Ultrasound biomicroscopy for the assessment of zonules after ocular trauma. Ophthalmology 2003;110:1340-43.

36. Genovesi-Ebert F, Rizzo S, Chiellini S, Gabbriellini G, Laddaga F, Nardi M. Ultrasound biomicroscopy in the assessment of secondary glaucoma after vitreoretinal surgery and silicone oil injection. Ophthalmologica 1998;212(Suppl)1:4-5.

37. Wei W, Yang W, Chen Z, Zhu X, Wang J. A study on ocular anterior segment structure after scleral buckling surgery for retinal detachment. Zhonghua Yan Ke Za Zhi 1999;35:309-11, 17.

38. Liu L, Wang T, Li Z. Studies of mechanism of malignant glaucoma using ultrasound biomicroscope. Zhonghua Yan Ke Za Zhi 1998;34:178-82, 10.

39. Wang T, Liu L, Li Z, Zhang S. Ultrasound biomicroscopic examination of secondary pupillary block glaucoma. Zhonghua Yan Ke Za Zhi 2000;36:413-5, 27.

40. Dada, et al.UBM in opaque grafts with postpenetrating glaucoma. Cornea 2008;27:402-05.

41. Matsui N, Kamao T, Azumi A. Case of metastatic intraocular malignant lymphoma with neovascular glaucoma. Nippon Ganka Gakkai Zasshi 2005;109:434-39.

42. Bhende M, Biswas J, Gopal L.Ultrasound biomicroscopy in the diagnosis and management of intraocular gnathostomiasis. Am J Ophthalmol 2005;140:140-42.

43. Gazzard G, Friedman DS, Devereux JG, Chew P, Seah SK. A prospective ultrasound biomicroscopy evaluation of changes in anterior segment morphology after laser iridotomy in Asian eyes. Ophthalmology 2003;110:630-38.

44. Breingan PJ, Esaki K, Ishikawa H, et al. Iridolenticular contact decreases following laser iridotomy for pigment dispersion syndrome. Arch Ophthalmol 1999;117:325-28.

45. Sakai H, Ishikawa H, Shinzato M, Nakamura Y, Sakai M, Sawaguchi S. Prevalence of ciliochoroidal effusion after prophylactic laser iridotomy. Am J Ophthalmol 2003;136:53738.

46. Dada T, Mohan S, Sihota R, Gupta R, Gupta V, Pandey RM. Comparison of ultrasound biomicroscopic parameters after laser iridotomy in eyes with primary angle closure and primary angle closure glaucoma. Eye 2007;21:956-61.

47. Kaushik S, Kumar S, Jain R, Bansal R, Pandav SS, Gupta A. Ultrasound biomicroscopic quantification of the change in anterior chamber angle following laser peripheral iridotomy in early chronic primary angle closure glaucoma Eye 2007;21:735-41.

48. Nonaka A, Kondo T, Kikuchi M, Yamashiro K, Fujihara M, Iwawaki T, Yamamoto K, Kurimoto Y. Cataract surgery for residual angle closure after peripheral laser iridotomy. Ophthalmology 2005;112:974-79.

49. Avitabile T, Russo V, Uva MG, Marino A, Castiglione F, Reibaldi A. Ultrasound-biomicroscopic evaluation of filtering blebs after laser suture lysis trabeculectomy.Ophthalmologica 1998;212 Suppl 1:17-21.

50. Khairy HA, Atta HR, Green FD, et al. Ultrasound biomicroscopy in deep sclerectomy. Eye 2005;19:555-60.
51. Contreras I, Noval S, Munoz-Negrete FJ. Ultrasound biomicroscopy in deep sclerectomy with a new acrylic implant Arch Soc Esp Oftalmol 2006;81:445-50.

52. Genovesi-Ebert F, Rizzo S, Chiellini S, Gabbriellini G, Laddaga F, Nardi M. Ultrasound biomicroscopy in the assessment of secondary glaucoma after vitreoretinal surgery and silicone oil injection. Ophthalmologica 1998;212(Suppl 1):4-5.

53. Park M, Tanito M, Nishikawa M, Chihara E. Ultrasound biomicroscopy of intrascleral lake after viscocanalostomy and cataract surgery. J Glaucoma 2004;13:472-78.

54. Canlas OA, Ishikawa H, Leibmann JM, et al. Ultrasound biomicroscopy before and after goniosynechialysis. Am J Ophthalmol 2001;132:570-71.

55. Dietlein TS, Engels BF, Jacobi PC, Krieglstein GK. UBM-guided chamber angle surgery for glaucoma management: An experimental study. Eye 2003;17:340-45.

56. Carrillo MM, Trope GE, Pavlin C, et al. Use of ultrasound biomicroscopy to diagnose Ahmed valve obstruction by iris. Can J Ophthalmol 2005;40:499-501.

57. Pereira FA, Cronemberger S. Ultrasound biomicroscopic study of anterior segment changes after phacoemulsification and foldable intraocular lens implantation. Ophthalmology 2003;110:1799806.

58. Sugimoto K, Ito K, Esaki K, Miyamura M, Sasoh M, Uji Y. Supraciliochoroidal fluid at an early stage after trabeculectomy. Jpn J Ophthalmol 2002;46:548-52.

59. Grigera D, Moreno C, Fava O, Girado SG. Ultrasound biomicroscopy in eyes with anterior chamber flattening after trabeculectomy. Can J Ophthalmol 2002;37:27-32; discussion 32-33.

60. Ochiai H, Chihara E, Chuman H, et al. Age and increased incidence of 'forward bowing' of the iris in normal eyes. J Glaucoma 1998;7:408-12.

61. Ishikawa H, Esaki K, Leibmann JM, et al. Ultrasound biomicroscopy dark room provocative testing: A quantitative method for estimating anterior chamber angle width. Jpn J Ophthalmol 1999;43:526-34.

62. Kaushik S, Jain R, Pandav SS, Gupta A. Evaluation of the anterior chamber angle in Asian Indian eyes by ultrasound biomicroscopy and gonioscopy. Indian J Ophthalmol 2006;54:159-63.

63. Spaeth GL, Azuara-Blanco A, Araujo SV, et al. Intraobserver and interobserver agreement in evaluating the anterior chamber angle configuration by ultrasound biomicroscopy. J Glaucoma 1997;6:13-17.

64. Radhakrishnan S, Goldsmith J, Huang D, et al. Comparison of optical coherence tomography and ultrasound biomicroscopy for detection of narrow anterior chamber angles. Arch Ophthalmol 2005;123:1053-59.

65. Dada T, Sihota R, Gadia R, Aggarwal A, Mandal S, Gupta V. Comparison of anterior segment optical coherence tomography and ultrasound biomicroscopy for assessment of the anterior segment. J Cataract Refract Surg 2007;33:837-40.

66. Kobayashi H, Kobayashi K, Kiryu, et al. Pilocarpine induces an increase in the anterior chamber anglular width in eyes with narrow angles. Br. J Ophthalmol 1999;83:553-58. 
67. Nissirios N, Ramos-Esteban J, Danias J. Ultrasound biomicroscopy of the rat eye: Effects of cholinergic and anticholinergic agents. Graefes Arch Clin Exp Ophthalmol 2005;243:469-73.

68. Marchini G, Ghilotti G, Bonadimani M, Babighian S. Effects of $0.005 \%$ latanoprost on ocular anterior structures and ciliary body thickness. J Glaucoma 2003;12:295-300.

69. Marraffa M, Marchini G, Pagliarusco A, et al. Ultrasound biomicroscopy and corneal endothelium in Nd:YAG-laser iridotomy. Ophthalmic Surg Lasers 1995;26:519-23.

70. Chiou AG, Mermoud A, Underdahl JP, et al. An Ultrasound biomicroscopic study of eyes after deep sclerectomy with collagen implant Ophthalmology 1998;105:746-50.

71. Aptel F, Dumas S, Denis P. Ultrasound biomicroscopy and optical coherence tomography imaging of filtering blebs after deep sclerectomy with new collagen implant. Eur J Ophthalmol 2009;19:223-30.

72. Mansouri K, Burgener ND, Bagnoud M, Shaarawy T. A prospective ultrasound biomicroscopy evaluation of changes in anterior segment morphology following laser iridotomy in European eyes. Eye. 2009 Jan 9 [Epub ahead of print].

73. Wu Q, Zhang Y, Song BW, Lu B, Guan JH. Evaluation of the bleb morphology and the function of postfiltration surgery using slit-lamp adapted optical coherence tomography and ultrasound biomicroscopy in glaucoma patients Zhonghua Yan Ke Za Zhi 2008;44:402-07.
74. Zhang Y, Wu Q, Zhang M, Song BW, DU XH, Lu B. Evaluating subconjunctival bleb function after trabeculectomy using slitlamp optical coherence tomography and ultrasound biomicroscopy. Chin Med J (Engl) 2008 20;121:1274-79.

75. Mansouri K, Sommerhalder J, Shaarawy T. Prospective comparison of ultrasound biomicroscopy and anterior segment optical coherence tomography for evaluation of anterior chamber dimensions in European eyes with primary angle closure. Eye 2009 May 15 [Epub ahead of print].

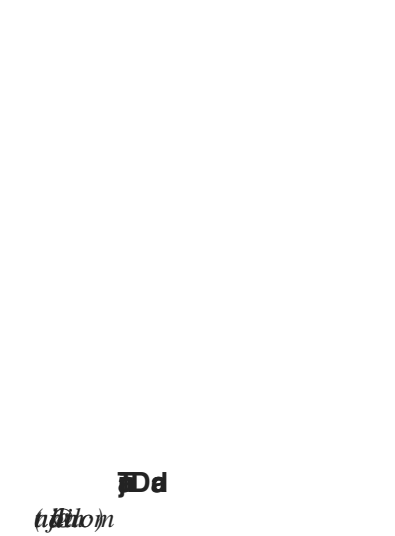

Those who deny freedom to others, deserve it not for themselves. 